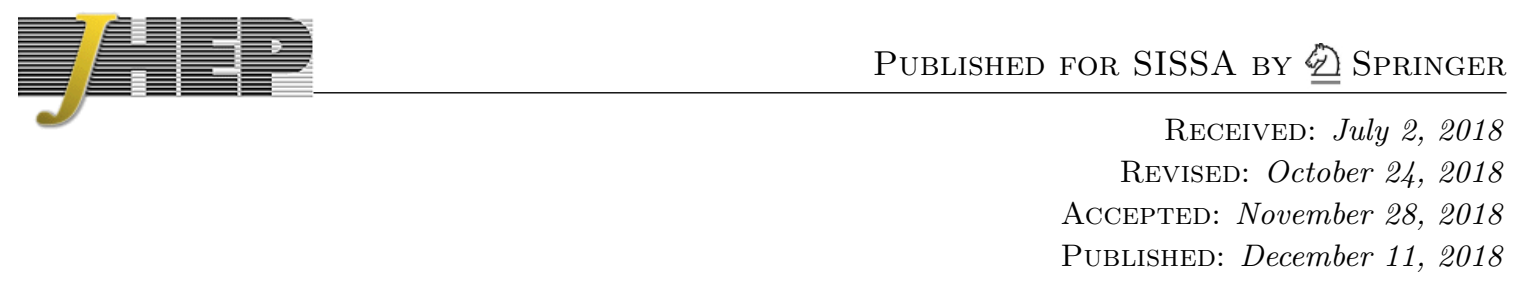

\title{
High-dimensional neutrino masses
}

\author{
Gaetana Anamiati, ${ }^{a}$ Oscar Castillo-Felisola, ${ }^{b, c}$ Renato M. Fonseca, ${ }^{d}$ J.C. Helo ${ }^{c, e}$ \\ and M. Hirsch ${ }^{a}$ \\ ${ }^{a}$ AHEP Group, Instituto de Física Corpuscular - CSIC/Universitat de València, \\ Edificio de Institutos de Paterna, Apartado 22085, E-46071 València, Spain \\ ${ }^{b}$ Universidad Técnica Federico Santa María, \\ Casilla 110-V, Valparaíso, Chile \\ ${ }^{c}$ Centro-Científico-Tecnológico de Valparaíso, \\ Casilla 110-V, Valparaiso, Chile \\ ${ }^{d}$ Institute of Particle and Nuclear Physics \\ Faculty of Mathematics and Physics, Charles University, \\ V Holešovičkách 2, 18000 Prague 8, Czech Republic \\ ${ }^{e}$ Departamento de Física, Facultad de Ciencias, Universidad de La Serena, \\ Avenida Cisternas 1200, La Serena, Chile \\ E-mail: anamiati@ific.uv.es, o.castillo.felisola@gmail.com, \\ fonseca@ipnp.mff.cuni.cz, jchelo@userena.cl, mahirsc@ific.uv.es
}

ABSTRACT: For Majorana neutrino masses the lowest dimensional operator possible is the Weinberg operator at $d=5$. Here we discuss the possibility that neutrino masses originate from higher dimensional operators. Specifically, we consider all tree-level decompositions of the $d=9, d=11$ and $d=13$ neutrino mass operators. With renormalizable interactions only, we find 18 topologies and 66 diagrams for $d=9$, and 92 topologies plus 504 diagrams at the $d=11$ level. At $d=13$ there are already 576 topologies and 4199 diagrams. However, among all these there are only very few genuine neutrino mass models: At $d=(9,11,13)$ we find only $(2,2,2)$ genuine diagrams and a total of $(2,2,6)$ models. Here, a model is considered genuine at level $d$ if it automatically forbids lower order neutrino masses without the use of additional symmetries. We also briefly discuss how neutrino masses and angles can be easily fitted in these high-dimensional models.

Keywords: Beyond Standard Model, Neutrino Physics

ARXIV EPRINT: 1806.07264 


\section{Contents}

1 Introduction 1

2 Preliminaries 3

2.1 Tree level $d=5$ and $d=7 \quad 3$

2.2 1-loop $d=5$ and $d=7$ diagrams 4

2.3 Genuineness 6

3 Classification and results $\quad 9$

3.1 Dimension $9(d=9) \quad 10$

$\begin{array}{lll}3.2 & \text { Dimension } 11(d=11) & 12\end{array}$

$\begin{array}{lll}3.3 & \text { Dimension } 13(d=13) & 13\end{array}$

3.4 A short discussion of phenomenological aspects 14

$\begin{array}{lll}3.4 .1 & \text { Running of gauge couplings } & 14\end{array}$

$\begin{array}{lll}3.4 .2 & \text { Collider physics } & 15\end{array}$

$\begin{array}{llr}4 & \text { Conclusions } & 18\end{array}$

$\begin{array}{ll}\text { A Neutrino mass and angle fits } & 19\end{array}$

B RGEs and $\beta$-coefficients for the different models 21

\section{Introduction}

The Weinberg operator is the lowest dimensional non-renormalizable operator that one can write down with only standard model (SM) fields [1]. It violates lepton number by two units and thus, once the electro-weak symmetry is broken, Majorana neutrino masses are generated. The observed smallness of the neutrino masses is then usually attributed to the large value of the scale of lepton number violation (LNV), typically $\Lambda \sim\left(10^{14}-10^{15}\right) \mathrm{GeV}$. This is the essence of the seesaw mechanism [2-12]. While simple and elegant, the large mass scale involved in this argument makes direct tests of the classical seesaw impossible.

There exist, however, many possibilities to explain the smallness of the observed neutrino masses with lower LNV scales. For Majorana neutrinos one can write in general [13]

$$
m_{\nu} \propto \epsilon \cdot\left(\frac{1}{16 \pi^{2}}\right)^{n} \cdot\left(\frac{v}{\Lambda}\right)^{d-5} \cdot \frac{v^{2}}{\Lambda} .
$$

Here, $v$ stands for the standard model vacuum expectation value (vev), $d$ is the dimension of the operator, $n$ stands for the number of loops at which neutrino masses are generated. $\epsilon$ expresses symbolically the additional suppression of lepton number violation that might 


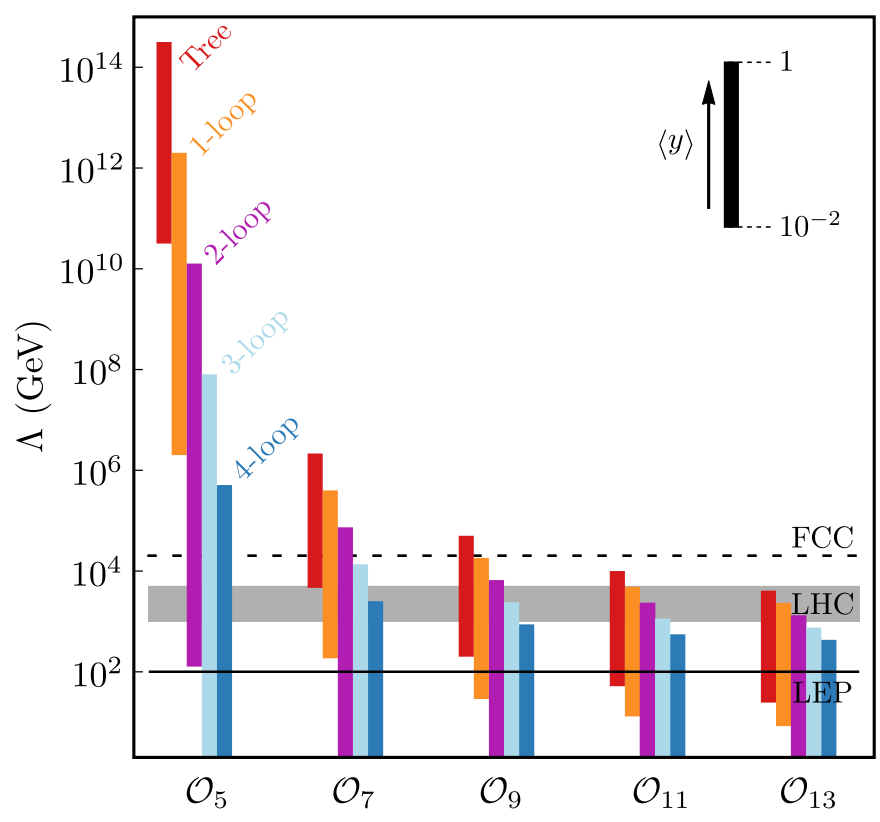

Figure 1. The typical energy scales $(\Lambda)$ for which a neutrino mass model with a given dimension and number of loops $(d, n)$ can explain correctly the observed sub-eV neutrino masses. Operators start at $d=5$, corresponding to the Weinberg operator. Energy ranges have been estimated using average couplings $\langle y\rangle$ in the range of $[0.01,1]$.

arise in particular constructions, such as for example the inverse seesaw mechanism [14]. Finally, in addition, small Yukawa or scalar couplings, not shown explicitly in eq. (1.1), could lead to smaller than expected neutrino masses.

Equation (1.1) can be used to estimate the typical scale $\Lambda$, for which the observed neutrino masses could be explained for a given $d$ and $n$. Figure 1 illustrates this estimate. Here, $\mathcal{O}_{5}$ at tree-level corresponds to the classical seesaw mechanism. Note that for $\mathcal{O}_{5}$ at tree-level (1-loop level) Yukawa couplings of order $\mathcal{O}\left(10^{-6}\right)\left(\mathcal{O}\left(10^{-3}\right)\right)$ would be needed to obtain a scale as low as $\Lambda \simeq 1 \mathrm{TeV}$. In this figure we also show the estimated reach for three colliders. The LEP line reflects that no electrically charged particle coupled to SM fermions with masses below roughly $100 \mathrm{GeV}$ can exist, after the negative searches performed at the LEP collider [15]. The horizontal grey band indicates a very rough estimate of the reach of the LHC: The lower edge of the band is a more conservative estimate (pair production of charged particles), while the upper edge is roughly the reach of the LHC for particles produced in s-channel diagrams and/or with colour. For $d=9$ and larger one expects that LHC experiments will cover an important part of the available parameter space of these models. We also show as a dashed line a rough estimate of the reach of a hypothetical $\sqrt{s}=100 \mathrm{TeV}$ collider, here called FCC. Thus, neutrino mass models generated at $d=9$ and higher should be testable in the near future. This simple argument forms the main motivation for our current paper.

Here, we will study high-dimensional tree-level diagrams for Majorana neutrino masses. We will treat systematically all possible topologies for the deconstruction of the $d=9$, 
$d=11$ and $d=13$ operators. We will identify all the "genuine" diagrams, which for us are those diagrams that can give the leading contribution to the neutrino mass matrix, without the use of extra (discrete or flavour) symmetries. We will discuss this requirement in more detail in section 2.3. Despite the large number of possible topologies, for $d=9$ and $d=11$ surprisingly only 4 models survive: 2 at $d=9$ and 2 at $d=11$. For $d=13$ we have found a total of 2 genuine diagrams and 6 models that can realize them.

Before presenting our analysis, let us briefly mention that, of course, many authors have studied neutrino mass models beyond the simplest tree-level seesaw, for a recent review see for example [16]. The Zee model [17], or the Zee-Babu model [11, 18, 19] are early examples of 1-loop and 2-loop realizations of the Weinberg operator. A systematic analysis of possible neutrino mass models at $d=5$ and 1-loop can be found in ref. [13], for a general analysis of $d=5$ models at 2-loop see ref. [20]. For the 3-loop case, there exist some well-known models in the literature [21, 22]; a complete study of 3-loop neutrino masses at $d=5$ can be found in ref. [23]. Neutrino masses at $d=7$ level have also been studied. A systematic analysis at tree-level was done in ref. [24]. At $d=7$ tree-level there is only one genuine (in our sense) tree-level neutrino mass model, which was first discussed in ref. [25]; we will call it the BNT model below. A general analysis of $d=7$ neutrino masses at 1-loop order was recently presented in refs. [26, 27].

Then there are also some papers on $d=9$ (and higher) neutrino mass models, see refs. [28-33]. We will come back to these papers briefly in section 2, where we discuss the main differences between their results and our present work. We mention in passing also the model presented in ref. [34], which uses a scalar septet to construct a model giving $d=13$ neutrino masses at 1-loop. Note, however, that this model is not genuine in our sense, since it uses a $Z_{2}$ symmetry to eliminate the $d=5$ seesaw contribution.

The rest of this paper is organized as follows. In section 2 we will set up our notation and briefly discuss neutrino mass generation at lower dimensions. This is necessary to clearly define what we mean by "genuine" models. Section 3 then contains the central piece of our work. We explain our methods, discuss topologies and list and briefly discuss the genuine models. In Section 4 we give a short conclusion. In the appendix we discuss how experimental data on neutrino masses and mixing can be easily fitted with these high-dimensional models.

\section{Preliminaries}

In this section we briefly go over some basic facts about $d=5$ and $d=7$ neutrino masses. This will be useful later, when we discuss genuine higher dimensional models, since those models can give the dominant contribution to the neutrino mass matrix only if $d=5$ and $d=7$ contributions are absent. We will use the following notation. A $\mathrm{SU}(2)_{L}$ multiplet with hypercharge $Y$ is denoted as $\mathbf{R}_{Y}$, to which we add the superscript $F$ or $S$ for fermion or scalar, respectively. Thus, for example $\mathbf{5}_{0}^{F}$ is a hypercharge-less fermionic quintuplet.

\subsection{Tree level $d=5$ and $d=7$}

The $d=5$ Weinberg operator can be generated at tree-level in exactly three different ways [35]. In the literature these are known as seesaw type-I, type-II and type-III. Type- 


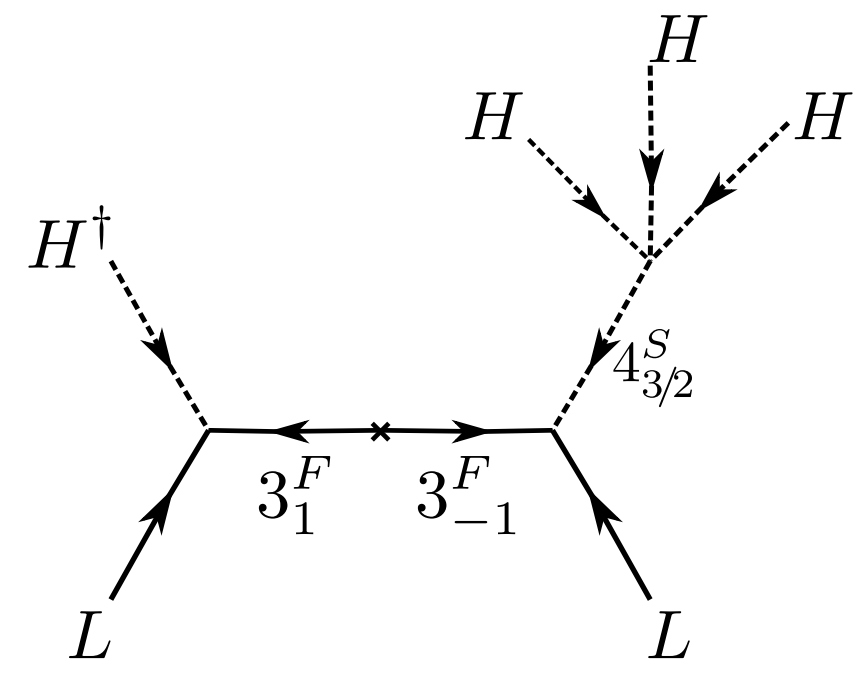

Figure 2. The genuine tree-level diagram for $d=7$ [25]. At least two beyond-the-SM particles are needed for higher dimensional operators.

I is the standard contribution due to right-handed neutrino $\nu_{R}$ (or $\mathbf{1}_{0}^{F}$ in our notation). The Majorana mass term for $\nu_{R}$ is the origin of lepton number violation. Type-III seesaw replaces $\mathbf{1}_{0}^{F}$ by $\mathbf{3}_{0}^{F}$ [12], which is a field usually denoted as $\Sigma$ in the literature. Finally, for type-II seesaw one introduces $\mathbf{3}_{1}^{S} \equiv \Delta$. In this latter case, the presence of both the Yukawa coupling $L \Delta L$ and the scalar coupling $H \Delta^{\dagger} H$, leads to lepton number violation.

At $d=7$ one already finds five different topologies [24]. However, one of these can not lead to any renormalizable neutrino mass model, while for three more topologies the diagrams always contain necessarily one of the $d=5$ seesaw mediators. The only diagram for which the $d=5$ tree-level seesaw is absent without the need of additional symmetries was first discussed in ref. [25]. This model contains two new particles, $\mathbf{3}_{1}^{F}$ and $\mathbf{4}_{3 / 2}^{S}$, as shown in figure 2 .

A few comments might be in order at this point. As figure 2 indicates, two Weyl fermions are actually needed to generate the diagram: $\mathbf{3}_{1}^{F}$ and its vector partner $\mathbf{3}_{-1}^{F}$. Without the mass term $m_{\mathbf{3}_{1}^{F}} \mathbf{3}_{1}^{F} \mathbf{3}_{-1}^{F}$ there would not be any source of lepton number violation in the model and, thus, no Majorana masses for the light, active neutrinos could be generated. We have therefore shown this mass insertion explicitly in figure 2. In many of the diagrams in the rest of this paper, on the other hand, for a more compact presentation, we do not explicitly show the vector partners. However, we stress that in all of our treelevel models all exotic fermions must necessarily be of vector-type or Majorana fermions. Also, while at $d=5$ one new particle is sufficient for each of the three seesaws, at $d=7$ we already need two different fields (three if one counts the vector fermion as two distinct Weyl fermions) for a genuine model.

\subsection{1-loop $d=5$ and $d=7$ diagrams}

The authors of ref. [13] systematically analyzed all 1-loop $d=5$ topologies. In total, there are 6 topologies, but only two of them (called T-1 and T-3) can yield genuine models in our 

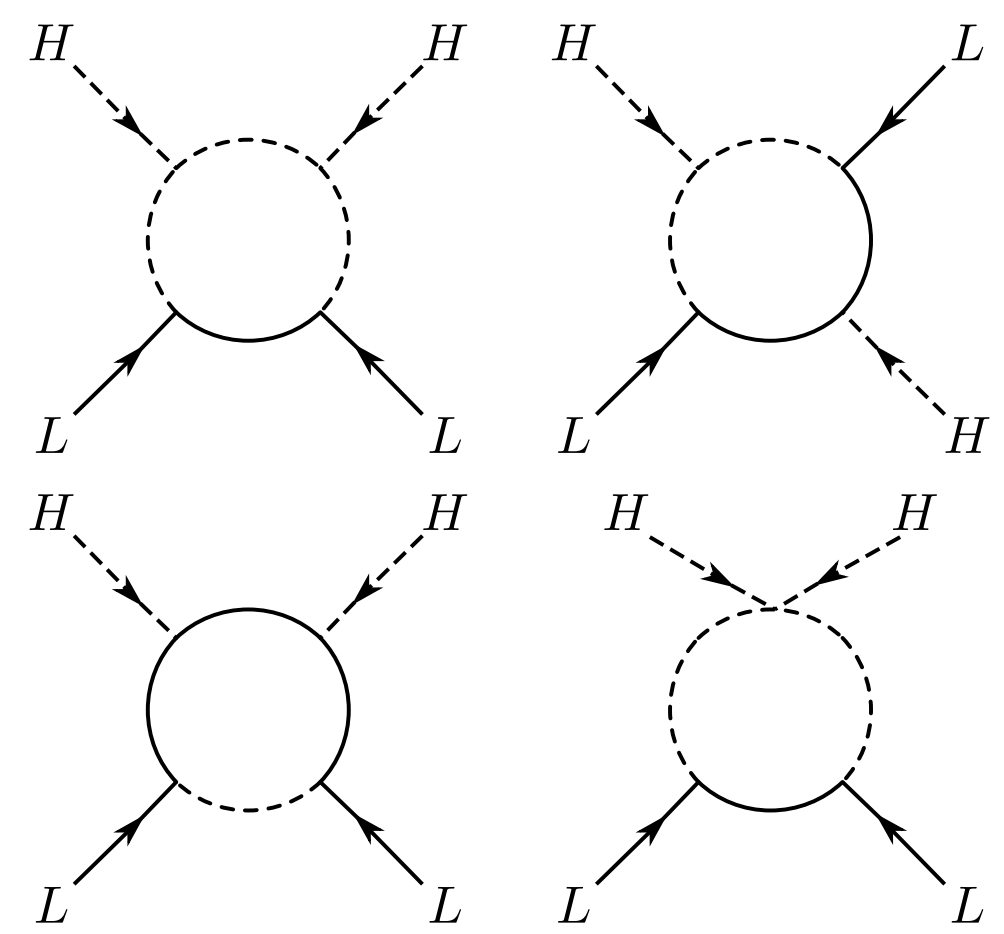

Figure 3. The four genuine 1-loop $d=5$ neutrino mass diagrams [13]. In the top panel we show the T-1-i diagram on the left and the T-1-ii diagram on the right. In the bottom panel, the T-1-iii diagram on the left and the T-3 diagram on the right.

sense. These lead to four different diagrams, shown in figure 3. T-1-ii corresponds to the diagram of the well-known Zee model [17], an example for T-3 is the scotogenic model [36]. Note also that in all 1-loop diagrams at least two beyond-the-SM fields are needed.

In contrast to tree-level diagrams, discussed above, for 1-loop diagrams the representation and hypercharges of the internal particles are not uniquely fixed. Since both $L$ and $H$ are $\mathrm{SU}(2)_{L}$ doublets, the two internal particles they meet in a trilinear vertex must transform as $(\mathbf{N})_{Y}^{S / F}$ and $(\mathbf{N}+\mathbf{1})_{Y \pm 1 / 2}^{S / F}$, for some unconstrained $\mathbf{N}$ and $Y .{ }^{1}$ This leads to a series of possible models at 1-loop, if one allows for larger $\mathrm{SU}(2)$ representations and hypercharges.

At $d=7$ 1-loop one finds already 48 different topologies, from which, however, only 8 can lead to genuine models [26]. The analysis of ref. [26] shows that there is only one diagram in which the largest internal representation can be as small as a triplet, while there are a further 22 diagrams, with at least one quadruplet. We will not repeat here all the diagrams for brevity and instead show in figure 4 just two examples.

Figure 4 shows on the left the model with only triplets, while the diagram on the right is an example of a model with exactly one quadruplet. The exotic particles external to

\footnotetext{
${ }^{1}$ Although less important for us, we mention that differently from the tree-level realizations, internal particles in the loops can also be coloured. In analogy to what happens for the SU(2) quantum numbers, since $L$ and $H$ are both colorless, the two internal particles which they meet in a trilinear vertex must transform as $\mathbf{R}$ and $\overline{\mathbf{R}}$ under $\mathrm{SU}(3)_{C}$, with $\mathbf{R}$ being arbitrary.
} 

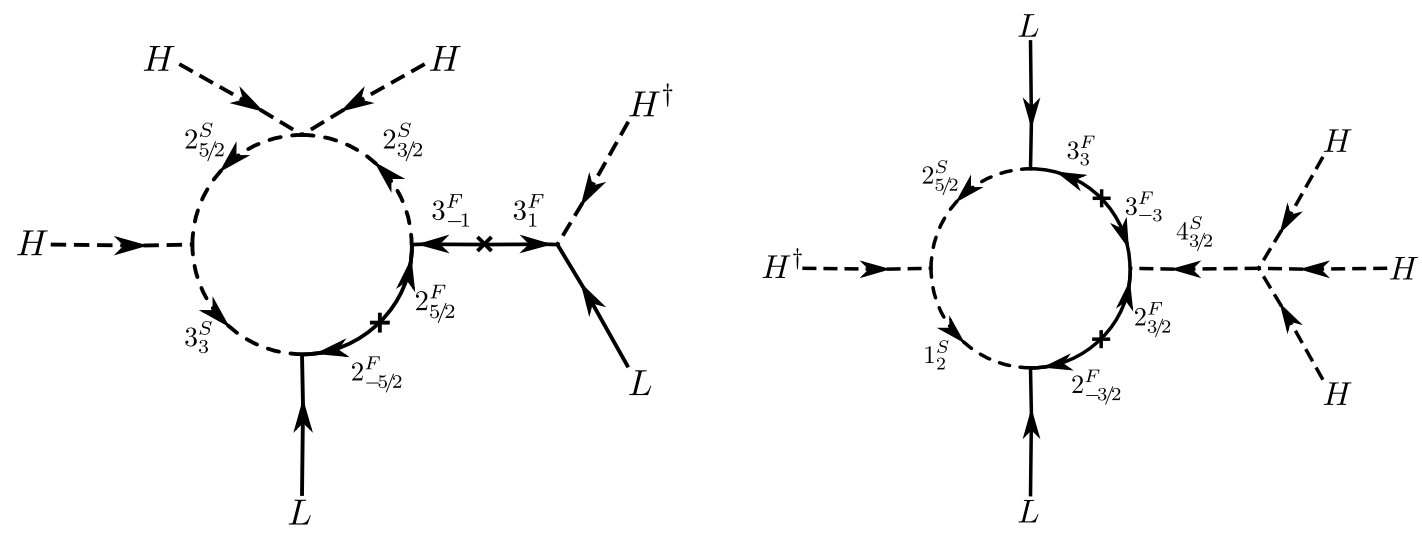

Figure 4. Two examples for genuine 1-loop $d=7$ diagrams. These examples have been chosen since they contain the smallest representations, for which genuine 1-loop $d=7$ diagrams can be constructed.

the loop in these example models are $\mathbf{3}_{1}^{F}$ (left diagram) and $\mathbf{4}_{3 / 2}^{S}$ (right diagram). These two particles can not be present in 1-loop $d=7$ models at the same time, otherwise one generates the $d=7$ tree-level diagram of the BNT model. Again, as in the case of $d=5$ 1-loop, one can build series of models allowing for larger representations and/or hypercharges for the particles in the loop. Also, from the fact that $H$ and $L$ have fixed quantum numbers, one can derive a set of conditions on the possible combinations of representations and hypercharges for the internal particles. The exact conditions, however, depend on the type of diagram under consideration. Note that most 1-loop $d=7$ diagrams need five new particles (more, if one counts the vector partners of the fermions as extra degrees of freedom), although for special values of the quantum numbers four new fields are enough.

\subsection{Genuineness}

In this subsection we want to discuss our concept of "genuineness" for neutrino mass models in somewhat more detail. In short, we consider a model genuine at dimension $d$, if all lower dimensional contributions are automatically absent, without the need for additional symmetries beyond those of the standard model group.

However, one aspect of higher-dimensional neutrino mass models needs to be considered first. There is a single $\Delta L=2$ neutrino mass operator of dimension $d$, which is always of the following form: ${ }^{2}$

$$
\mathcal{O}^{d} \propto L L H H\left(H^{\dagger} H\right)^{(d-5) / 2},
$$

\footnotetext{
${ }^{2}$ Invariance under $\mathrm{SU}(2)$ forces the operator dimension $d$ to be odd. Note also that $\mathcal{O}^{d}$ in eq. (2.1) contains $d-1 \equiv 2 n$ doublets of $\mathrm{SU}(2)$, and in general the product of multiple doublets is expected to have many independent contractions. Indeed, it seems that the number of singlets in the product of $2 n$ doublets is given by the Catalan numbers $C(n) \equiv(2 n) ! /[(n+1) ! n !]$, hence for $d=13$ (i.e. $n=6$ ) we might had expected 132 different contractions of the $\mathrm{SU}(2)$ indices. Yet, we note that there is a single Higgs field, so all $(d-1) / 2$ copies of $H$ must be contracted symmetrically; the same is true for the $(d-5) / 2$ copies of $H^{*}$. And it is not complicated to see that for a given $d$, there is always one - and only one - contraction with this property; all the others are therefore identically 0. See also ref. [37].
} 
with the $\mathrm{SU}(2)$ indices of each pair $L H$ outside the brackets contracted with the antisymmetric real tensor $\epsilon_{a b}$, and each pair $H^{\dagger} H$ inside the brackets contracted with the $\delta_{a b}$ tensor.

The very same operators will always lead to lower order loop models: ${ }^{3}$

$$
\frac{1}{\Lambda^{(d-4)}} L L H H\left(H^{\dagger} H\right)^{(d-5) / 2} \rightarrow \frac{1}{16 \pi^{2}} \frac{1}{\Lambda^{(d-6)}} L L H H\left(H^{\dagger} H\right)^{(d-7) / 2}
$$

In the SM, where there is only one Higgs doublet, such loops can not be forbidden by postulating some symmetry. ${ }^{4}$ One can straightforwardly estimate that such a loop contribution will become more important than the tree-level one if $(\Lambda / v) \gtrsim 4 \pi$. This means $\Lambda \lesssim 2 \mathrm{TeV}$ is required for the $d$-dimensional tree-level contribution to dominate over the $(d-2)$ dimensional 1-loop one. Since this is unavoidable in the SM, $d \geq 7$ tree-level model of neutrino mass must have new particles below $2 \mathrm{TeV}$, otherwise loop contributions will dominate the neutrino mass matrix. Note that this "upper limit" is more stringent than the estimates for the typical scales $\Lambda$ shown in figure 1 .

In loop calculations usually there appear both finite and infinite loop integrals. However, in a renormalizable theory, infinite contributions are canceled by counter-terms, implying that there are lower order contributions to the same operator. Thus, all models with diagrams requiring renormalization are not genuine in our sense. On the other hand, diagrams associated to finite loop integrals only, can lead to genuine models. One should distinguish two different scenarios: Models in which lower order contributions are absent automatically, and models which forbid lower order contributions with the help of an extra symmetry. We consider only the former class of models genuine.

Let us discuss the second scenario with one concrete and well-known example: the scotogenic model [36]. Here, the right-handed neutrino is assumed to be odd under a $Z_{2}$ and a new scalar doublet (odd under the $Z_{2}$ as well) is added to the model. Thus, there is no $d=5$ tree-level contribution from the SM Higgs and the 1-loop contribution can dominate. The resulting 1-loop integral is finite and thus, technically, no tree-level neutrino mass term is needed. Let us stress that while we do not consider such a construction to be "genuine" in our sense, such neutrino mass models are of course perfectly valid and phenomenologically interesting models.

However, we also want to mention that such a construction relies on the assumption that the new scalars in these models do not acquire a vacuum expectation values. Of course, adding some discrete symmetry to the model does not guarantee, by itself, the absence of a vev. Rather, a non-zero vev for the exotic scalar(s) would break the discrete symmetry spontaneously, leading to an unwanted tree-level neutrino mass term and thus usually (but not always) vevs are to be avoided. This can be achieved with an appropriate choice of parameter values in the scalar potential. (In the scotogenic model essentially it

\footnotetext{
${ }^{3}$ Diagrammatically, this does not mean that one can close every pair of $H, H^{*}$ external lines. However, there will always be at least one such pairs of lines which can be closed.

${ }^{4}$ For this reason, the authors of ref. [24] considered a two-Higgs doublet extension of the SM. Assigning different charges to the two doublets under a new $Z_{n}$ makes it possible to forbid loop contributions. Note, however, that these additional symmetries are spontaneously broken by the doublet vevs.
} 

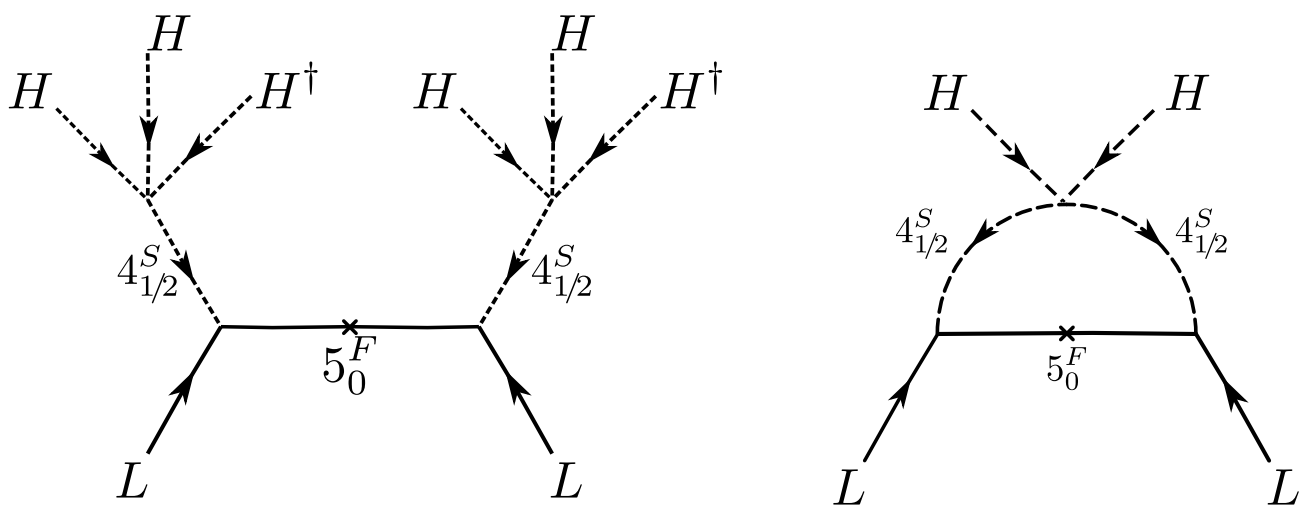

Figure 5. One example of a "non-genuine" $d=9$ diagram, to the left. Connecting the two quadruplet scalars, as shown on the right, leads to a 1-loop diagram at $d=5$.

corresponds to imposing the condition that the mass squared parameter of the new scalar doublet is positive, $\mu_{D}^{2}>0$.)

For concreteness, let us now consider a particular example model for a $d=9$ tree-level diagram. The model presented in ref. [29] contains two new fields: $\mathbf{4}_{1 / 2}^{S}$ and $\mathbf{5}_{0}^{F}$. This model is non-genuine in our definition. ${ }^{5}$ The model generates a $d=9$ tree-level diagram, see figure 5 on the left, via the four scalar vertex $\lambda_{4}\left(4_{1 / 2}^{S}\right)^{\dagger} H H H^{\dagger} .6$ Connecting the two quadruplet scalars via a quartic interaction $\lambda_{5}\left(\mathbf{4}_{1 / 2}^{S}\right)^{\dagger}\left(\mathbf{4}_{1 / 2}^{S}\right)^{\dagger} H H$ allows one to draw the 1-loop $d=5$ diagram on the right. The loop integral is finite, just as in the scotogenic model. Assuming the masses of $\mathbf{4}_{1 / 2}^{S}$ and $\mathbf{5}_{0}^{F}$ to be roughly of order $\Lambda$ the ratio of the contributions of the two diagrams can be estimated as

$$
R(\text { tree/loop }) \propto \frac{\lambda_{4}^{2}}{\lambda_{5}}\left(\frac{v_{S M}}{\Lambda}\right)^{4}
$$

i.e. the tree-level will be less important than the loop for scales $\Lambda$ bigger than roughly $\Lambda \simeq 600 \sqrt{\lambda_{4}} /\left(\lambda_{5}\right)^{1 / 4} \mathrm{GeV}$. Note that, since $\mathbf{4}_{1 / 2}^{S}$ contains one doubly charged component, the LHC searches on same-sign dileptons [38] should apply. Thus, one can estimate that the current lower limits on the mass of $\mathbf{4}_{++}^{S}$ should be in the range of roughly $[500,650]$ $\mathrm{GeV}$, depending on the final state lepton generation [27].

Similar comments apply to the models presented in refs. [28, 30-32]. Reference [28] introduces $\mathbf{5}_{1}^{F}, \mathbf{4}_{3 / 2}^{S}$ and $\boldsymbol{4}_{1 / 2}^{S}$. The model has a 1-loop diagram of type $T_{3}$, just as in the example of ref. [29] discussed above. Reference [30] introduces the idea of a "cascade seesaw". Essentially here the author discusses that models such as [29] can be generalized to yield $d=9, d=13$ and higher, by using larger and larger multiplets. References [31, 32] discusses different seesaw models at $d=7$ and $d=9$. However, this analysis considers only one exotic fermion (and two new scalars) in each model. None of the models in refs. [31, 32] is genuine in our sense.

\footnotetext{
${ }^{5}$ The same model was discussed also in ref. [33].

${ }^{6} \lambda_{4}$ will lead to an induced vev for $4_{1 / 2}^{S}$ even if the mass squared parameter $m_{S_{4}}^{2}$ is positive.
} 


\section{Classification and results}

The basic steps in the procedure are similar for $d=9, d=11$ and $d=13$. At each $d$ we first generate all possible topologies via a computer code based on known algorithms - for clear and self-contained explanations on how this can be done see [39, 40]. Here, we will only mention briefly the main idea. What we call a topology is what is known as a (undirected) graph to mathematicians, and it consists on some number of vertices connected among themselves by (undirected) edges. There is the obvious way of iteratively generating all graphs with $n$ vertices by adding one vertex to all graphs with one less vertex, in all possible ways. However, this procedure generates many equivalent/isomorphic topologies. Checking whether two graphs are isomorphic is a well known problem, which can be time consuming. In order to avoid doing these checks as much as possible, one can instead use a more targeted recipe which generates all graphs with a given degree sequence (the degree of a vertex $i$ is the number of edges connected to it, and the ordered sequence of numbers $d_{i}$ constitutes the graph's degree sequence; for example the topologies in figure 5 have degree sequences 443311111111 and 4331111). We used this latter approach [39, 40], even though a more naive one would probably also be feasible for the rather simple, tree-topologies under consideration.

Once these are obtained, we find all diagrams simply by labeling each line as a fermion or a scalar in all possible ways, and ensuring that one obtains fermion-fermion-scalar, scalar-scalar-scalar and scalar four-point vertices only. From these (large) lists of diagrams one can construct all models by searching for every allowed combination of $L, H$ and $H^{\dagger}$ in the outer legs of the diagrams.

From these lists we then eliminate every model, which is non-genuine in our definition. This is achieved in several steps, many of which can be automatized (we have written a Mathematica code for this purpose). The correctness of this code was cross-checked by visual inspection for all $d=9$ and $d=11$ models. For $d=13$ we cross-checked by hand that the models that were listed as genuine by the code did indeed not lead to lower dimensional neutrino masses. The basic idea behind this code is the following. Assume, for example, that one starts with excluding models which do lead to tree-level neutrino masses at $d=5$. One could simply cross from the list of models all those that contain the fields $\mathbf{1}_{0}^{F}, \mathbf{3}_{0}^{F}$ or $\mathbf{3}_{1}^{S}$. To do so, however, it is not necessary to calculate all the quantum numbers of the fields inside the diagrams. It is sufficient to realize that, for example, if a combination of fields such as $H L$ or $L L$ appear at the extreme end of a diagram, this is equivalent to the existence of $\mathbf{1}_{0}^{F} / \mathbf{3}_{0}^{F}$ or $\mathbf{3}_{1}^{S}$ in the diagram. Similarly, all conditions for $d=7$ and higher (and also for loops) can be reformulated as a search for combination of fields that form certain groups after cuts into the diagrams. We have found that programming these cuts is simpler than calculating the quantum numbers for all fields and then eliminating unwanted fields on a case-by-case basis.

We will not show all possible topologies and diagrams here for brevity. However, the complete lists can be found at renatofonseca.net/high-dim-neutrino-masses.php. 


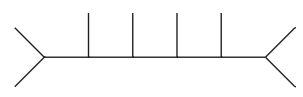

$T_{1}$

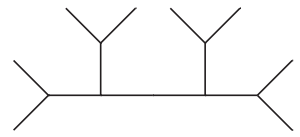

$T_{4}$

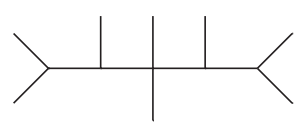

$T_{7}$

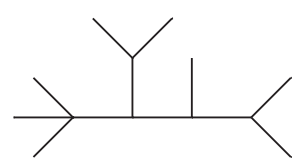

$T_{10}$

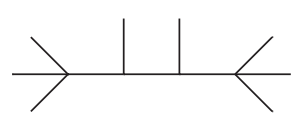

$T_{13}$

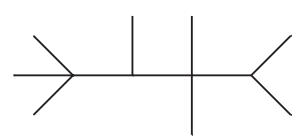

$T_{16}$

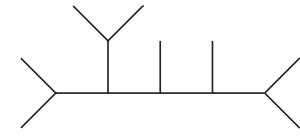

$T_{2}$

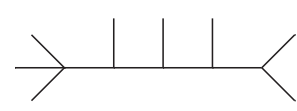

$T_{5}$

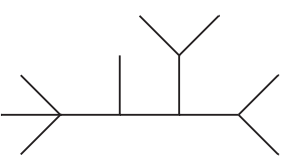

$T_{8}$

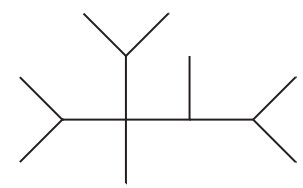

$T_{11}$

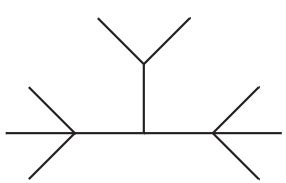

$T_{14}$

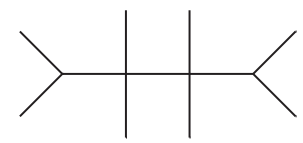

$T_{17}$

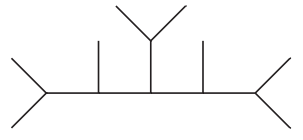

$T_{3}$

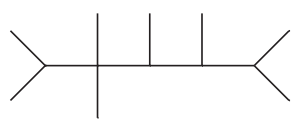

$T_{6}$

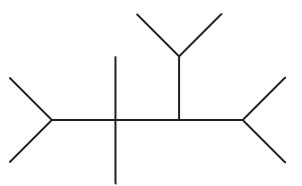

$T_{9}$

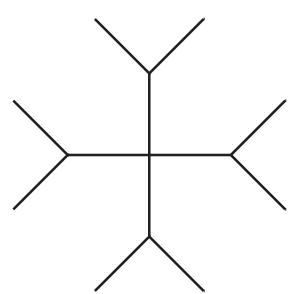

$T_{12}$

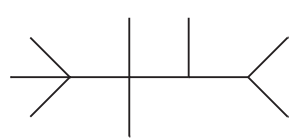

$T_{15}$

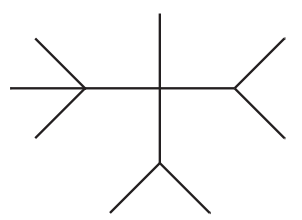

$T_{18}$

Figure 6. The 18 topologies at $d=9$ level that can give renormalizable diagrams.

\subsection{Dimension $9(d=9)$}

We start the discussion with $d=9$. Figure 6 shows all 18 topologies from which one can build valid neutrino mass diagrams with renormalizable vertices only. There is one more topology (not shown), with 8 external lines and no loops, but it requires three 4-point vertices, hence it will lead only to non-renormalizable models. The 18 topologies which we do show generate a total of 66 diagrams. However, all except four topologies lead only 

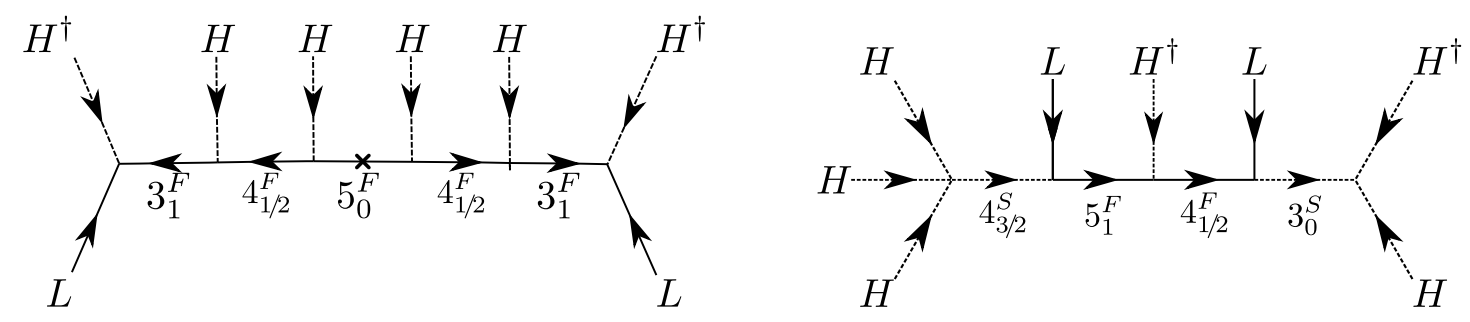

Figure 7. The two genuine diagrams at $d=9$.

to diagrams that necessarily have a tree-level neutrino mass at either $d=5$ or $d=7$. Diagrams from two more topologies will always also generate 1-loop $d=5$ diagrams hence, in the end, only topologies $T_{1}$ and $T_{5}$ yield diagrams that are genuine in our sense. But not all diagrams obtained from $T_{1}$ and $T_{5}$ are genuine either; the only ones which are genuine can be seen in figure 7 .

Consider first the diagram on the left hand side of figure 7. It contains only three new fermions, $\mathbf{3}_{1}^{F}, \mathbf{4}_{1 / 2}^{F}$ and $\mathbf{5}_{0}^{F}$, together with their vector partners, and no exotic scalar. This is the minimal genuine model at $d=9$. Its Lagrangian is given by:

$$
\mathcal{L}=\mathcal{L}_{\mathrm{SM}}+\mathcal{L}_{\text {Yuk }}+\mathcal{L}_{\text {mass }}
$$

where

$$
\begin{aligned}
\mathcal{L}_{\text {Yuk }}= & Y_{\nu} L \cdot \mathbf{3}_{1}^{F} H^{\dagger}+\bar{Y}_{\mathbf{3 4}} \mathbf{3}_{-1}^{F} \cdot \mathbf{4}_{1 / 2}^{F} H+Y_{\mathbf{3 4}} \mathbf{3}_{1}^{F} \cdot \mathbf{4}_{-1 / 2}^{F} H^{\dagger} \\
& +\bar{Y}_{\mathbf{4 5}} \mathbf{4}_{1 / 2}^{F} \cdot \mathbf{5}_{0}^{F} H^{\dagger}+Y_{\mathbf{4 5}} \mathbf{4}_{-1 / 2}^{F} \cdot \mathbf{5}_{0}^{F} H
\end{aligned}
$$

and

$$
\mathcal{L}_{\text {mass }}=M_{\mathbf{3}} \mathbf{3}_{1}^{F} \mathbf{3}_{-1}^{F}+M_{\mathbf{4}} \mathbf{4}_{1 / 2}^{F} \mathbf{4}_{-1 / 2}^{F}+M_{\mathbf{5}} \mathbf{5}_{0}^{F} \mathbf{5}_{0}^{F} .
$$

The light neutrino mass can be estimated in seesaw approximation (for one generation) as:

$$
m_{\nu} \simeq Y_{\nu}^{2} \bar{Y}_{\mathbf{3 4}}^{2} Y_{\mathbf{4 5}}^{2} \frac{v_{\mathrm{SM}}^{6}}{M_{\mathbf{3}}^{2} M_{\mathbf{4}}^{2} M_{\mathbf{5}}}
$$

For masses of the order of $\mathcal{O}(1-2) \mathrm{TeV}$, Yukawas of the order of $(0.03-0.04)$ will reproduce the scale of the atmospheric neutrinos, $m_{\nu} \simeq \sqrt{\Delta\left(m_{\text {Atm }}^{2}\right)} \simeq 0.05 \mathrm{eV}$. For a more detailed fit of neutrino masses and angles, see the appendix.

The diagram on the right hand side of figure 7 contains two exotic scalars and two exotic fermions. We give only the part of the Lagrangian relevant for the calculation of the neutrino mass,

$$
\begin{aligned}
\mathcal{L} \propto & \lambda_{4} H H H\left(\mathbf{4}_{3 / 2}^{S}\right)^{\dagger}+\mu_{3} \mathbf{3}_{0}^{S} H H^{\dagger}+m_{4}^{2}\left|\mathbf{4}_{3 / 2}^{S}\right|^{2}+m_{3}^{2}\left|\mathbf{3}_{0}^{S}\right|^{2} \\
& +Y_{5 L} L \cdot \mathbf{5}_{-1}^{F} \mathbf{4}_{3 / 2}^{S}+Y_{45} \mathbf{4}_{-1 / 2}^{F} \cdot \mathbf{5}_{1}^{F} H^{\dagger}+Y_{4 L} L \cdot \mathbf{4}_{1 / 2}^{F}\left(\mathbf{3}_{0}^{S}\right)^{\dagger} \\
& +M_{\mathbf{5}_{\mathbf{1}}} \mathbf{5}_{-1}^{F} \mathbf{5}_{1}^{F}+M_{\mathbf{4}} \mathbf{4}_{-1 / 2}^{F} \mathbf{4}_{1 / 2}^{F} .
\end{aligned}
$$

Again in seesaw approximation and for one generation we can roughly estimate the size of the neutrino mass generated by this model as,

$$
m_{\nu} \simeq Y_{5 L} Y_{4 L} Y_{45} \lambda_{4} \frac{\mu_{3} v_{S M}^{6}}{M_{\mathbf{5}_{\mathbf{1}}} M_{\mathbf{4}} m_{4}^{2} m_{3}^{2}}
$$



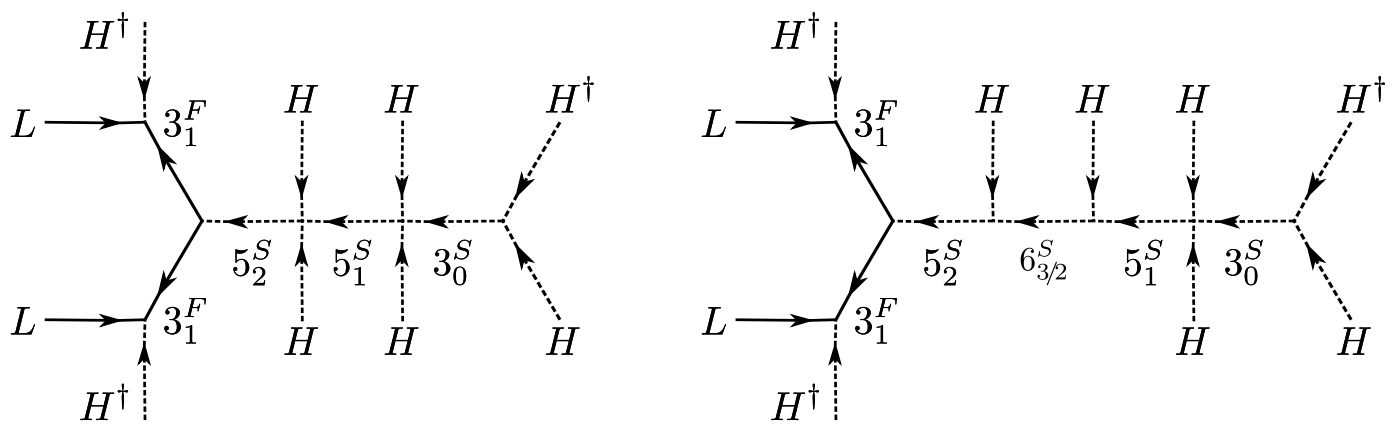

Figure 8. The two genuine diagrams at $d=11$. Note that the fields on the right-hand side diagram always produce the diagram on the left-hand side.

With all mass parameters equal to $1 \mathrm{TeV}, \mu_{3}=M_{\mathbf{5}_{\mathbf{1}}}=M_{\mathbf{4}}=m_{4}=m_{3}=1 \mathrm{TeV}$, and for $\lambda_{4}=Y_{5 L}=Y_{4 L}=Y_{45}=\mathcal{O}\left(10^{-2}\right)$ this gives roughly $0.3 \mathrm{eV}$. A more detailed description on how all neutrino data can be fitted in this model is deferred to the appendix.

\subsection{Dimension $11(d=11)$}

At $d=11$ we find 92 topologies, which generate a total of 504 diagrams. It is not very instructive to discuss in detail all the topologies and diagrams, as the methodology for eliminating non-genuine models is the same as for the $d=9$ case. The only two genuine diagrams are shown in figure 8 , and they are based on very similar models. The diagram on the right contains five new particles, four of which are also present in the diagram on the left. Thus, the model for the right diagram always produces also the diagram on the left. Unsurprisingly, at $d=11$ genuine diagrams require at least four different beyond-SM particles and large representations: At least two different quintuplets are needed and the model shown in the right diagram of figure 8 requires in addition a sextuplet.

Again, we write down only the part of the Lagrangian relevant for estimating the neutrino mass,

$$
\begin{aligned}
\mathcal{L} \propto & \lambda_{55} \mathbf{5}_{-2}^{S} \mathbf{5}_{1}^{S} H H+\lambda_{53} \mathbf{5}_{-1}^{S} \mathbf{3}_{0}^{S} H H+\mu_{61} \mathbf{5}_{1}^{S} \mathbf{6}_{-3 / 2}^{S} H+\mu_{62} \mathbf{5}_{-2}^{S} \mathbf{6}_{3 / 2}^{S} H \\
& +\mu_{3} \mathbf{3}_{0}^{S} H H^{\dagger}+m_{3}^{2}\left|\mathbf{3}_{0}^{S}\right|^{2}+m_{5_{1}}^{2}\left|\mathbf{5}_{1}^{S}\right|^{2}+m_{5_{2}}^{2}\left|\boldsymbol{5}_{2}^{S}\right|^{2}+m_{6}^{2}\left|\mathbf{6}_{3 / 2}^{S}\right|^{2} \\
& +Y_{\nu} L \cdot \mathbf{3}_{1}^{F} H^{\dagger}+Y_{3} \mathbf{3}_{-1}^{F} \cdot \mathbf{3}_{-1}^{F} \mathbf{5}_{2}^{S}+M_{3} \mathbf{3}_{-1}^{F} \mathbf{3}_{1}^{F}
\end{aligned}
$$

A simple estimate for the neutrino mass from the left diagram in figure 8 gives:

$$
m_{\nu} \simeq Y_{\nu}^{2} Y_{3} \lambda_{55} \lambda_{53} \frac{\mu_{3} v_{S M}^{8}}{M_{3}^{2} m_{3}^{2} m_{5_{2}}^{2} m_{5_{2}}^{2}}
$$

For a new physics scale of $\Lambda=1 \mathrm{TeV}$ and all dimensionless couplings order 0.05 one finds again a neutrino mass of order $0.3 \mathrm{eV}$. As is also the case for the second of our $d=9$ models, the dimensionful scalar coupling $\mu_{3}$ can be a source of additional neutrino mass suppression. For $\mu \simeq 100 \mathrm{keV}$ and all dimensionless couplings $\mathcal{O}(1)(\Lambda=1 \mathrm{TeV})$ one finds $m_{\nu} \sim \mathcal{O}(0.1) \mathrm{eV}$. 

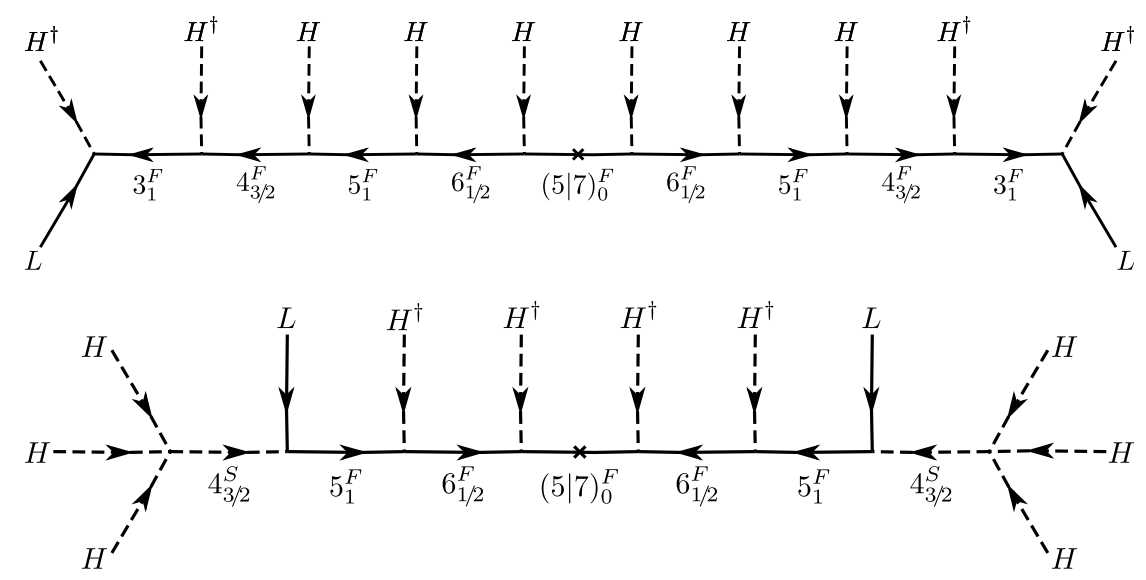

Figure 9. The two genuine diagrams at $d=13$ can be realized in a total of 6 models. It is possible to have either a $\mathbf{5}_{0}^{F}$ or a $\mathbf{7}_{0}^{F}$ in the middle. Furthermore, two extra models are obtained from the top diagram by rearranging appropriately the external $H^{\prime}$ 's and $H^{\dagger}$ 's and replacing either one or both of the internal $\boldsymbol{4}_{3 / 2}^{F}$ correspondingly by a $\boldsymbol{4}_{1 / 2}^{F}$. Note also that these two extra models (not represented above) with the field $\boldsymbol{4}_{1 / 2}^{F}$ need $\boldsymbol{7}_{0}^{F}$ in the middle of the diagram in order to be genuine.

A straightforward calculation shows that the diagram on the right gives a neutrino mass of roughly the same numerical value, if $\left(\mu_{61} \mu_{62}\right) / m_{6}^{2} \simeq \lambda_{55}$. Thus, the diagram on the left is the dominant one, if either $\mu_{61}$ or $\mu_{62}$ (or both) are very small relative to the new physics scale $\Lambda$. On the other hand, for $\lambda_{55} \ll 1$ the opposite situation is found.

\subsection{Dimension $13(d=13)$}

At $d=13$ there are 576 topologies and 4199 diagrams. One has to delete not only all models that lead to a tree level $d=5, d=7, d=9$ and $d=11$ diagram, as well as a 1-loop $d=5$ or $d=7$ diagram, but also all models with a 1-loop $d=9$ diagram. The last cut drastically reduces again the list of genuine models: without it nearly 50 different diagrams remain, while after this cut only 2 genuine tree-level diagrams at $d=13$ remain.

Figure 9 shows the two remaining genuine diagrams. Unsurprisingly, more fields and larger representations are needed in these diagrams. The largest representation is now a $\mathrm{SU}(2)_{L}$ septet. There is a total of six model variations that one can find for these two diagrams. In addition to the four models shown, one can construct two more model variations for the first diagram (top row): Replace either one or both of the $\mathbf{4}_{3 / 2}^{F}$ by a $\mathbf{4}_{1 / 2}^{F}$ (rearranging $H$ and $H^{\dagger}$ correspondingly). Note that the models with $\mathbf{4}_{1 / 2}^{F}$ are only genuine with a $\mathbf{7}_{0}^{F}$.

Let us discuss first briefly the models corresponding to the diagram in the top row. These models contain only new fermions, but no exotic scalars, and are very similar to each other. The models shown contain five new fermions. As mentioned above, there are two more variations containing a $\mathbf{4}_{1 / 2}^{F}$. Comparing these fermion-only models with the simplest $d=9$ model, one sees that higher dimensional fermion-only diagrams ( $d=17,21$ etc.) could be straightforwardly found, following the same construction principles. 
We will not write down the complete Lagrangian for these $d=13$ models for brevity. The neutrino mass is estimated for these models to be of order $m_{\nu} \simeq Y^{10} \frac{v^{10}}{\Lambda^{9}}$, where $Y$ stands symbolically for the Yukawa couplings in the diagrams and we assumed for simplicity that all masses are of order $\Lambda$. Yukawa couplings now have to be of order $\mathcal{O}(0.3)$ (with $\Lambda=1 \mathrm{TeV})$ for a neutrino mass $m_{\nu} \simeq(0.1-0.2) \mathrm{eV}$.

The remaining $d=13$ models in the bottom row of figure 9 need four exotic fields, one of them needs to be an exotic scalar. Again, a fermionic septet is the largest $\mathrm{SU}(2)_{L}$ representation. Since in these models, some of the Yukawa couplings from the fermiononly models are replaced by four-point scalar couplings, slightly smaller couplings, say $\mathcal{O}(0.2)$, are needed here to achieve $m_{\nu} \simeq(0.1-0.2) \mathrm{eV}$. We close this subsection by stating again that all $d=13$ models can easily fit all measured neutrino mass squared differences and angles.

\subsection{A short discussion of phenomenological aspects}

In this paper we are mostly concerned in classifying neutrino mass models. For completeness, however, in this section we add a brief discussion of the phenomenology of these models. For brevity, we will focus on only two aspects. First we discuss the running of the gauge couplings. Then, we turn to collider physics.

\subsubsection{Running of gauge couplings}

A common feature of all genuine tree-level neutrino models identified in the previous sections is that large $\mathrm{SU}(2)_{L}$ multiplets are required in order to avoid lower dimensional contributions to the neutrino mass matrix. Adding new $\mathrm{SU}(2)_{L}$ multiplets to the standard model changes the running of the gauge couplings. Since our tree-level models do not add any beyond the standard model (BSM) coloured fields, the value of the $\mathrm{SU}(3)_{c}$ coupling constant is not affected.

Figure 10 shows the running of the inverse of $g_{2}^{2}$ as a function of energy for six different models. These are the two +two genuine models with $d=9$ and $d=11$, plus two more with $d=13$. Note that we found six $d=13$ genuine neutrino mass models: the two considered in figure 10 contain the multiplets $5_{F}^{0}$ (the variants with $7_{F}^{0}$ exhibit an even stronger running of $g_{2}$ ). In appendix B we give the $\beta$ coefficients at 1-loop and 2-loop for these models. The numerical results shown in figure 10 assume that the new states have all masses of roughly $m \sim 2 \mathrm{TeV}$. For each model we show two different cases: (i) the running when only one copy of the exotic fermions is present and (ii) with three copies of exotic fermions. In all cases we use only one copy for the exotic scalars. This choice is motivated by the fact that standard model fermions come in three generations, but the neutrino mass fit in several of the models could be done with only one copy of the exotic fermions. Running is stronger, of course, with more fermions. Thus, one can understand our choice as representing the extreme cases, that could be realized.

As the figure shows, for all models Landau poles appear in $g_{2}$ below the grand unification scale (GUT). This affirms again our supposition that all these high dimensional neutrino mass models really have to be considered as low-energy constructions. The energy at which the Landau poles appear varies strongly from model to model. For $d=13$ and 


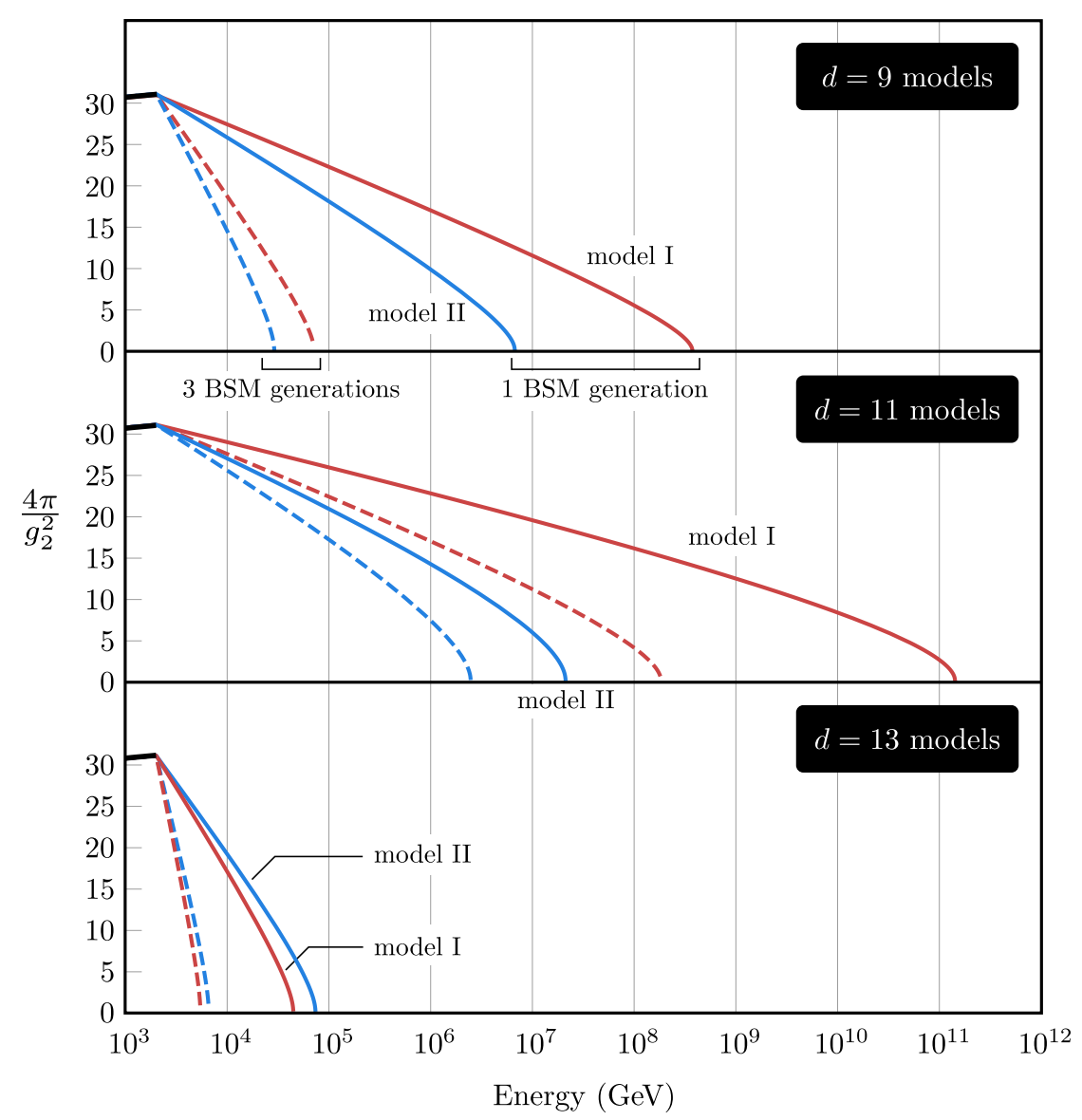

Figure 10. Running of the (inverse of) the gauge coupling $g_{2}$ for six of the eight different genuine neutrino mass models we have found: two at $d=9$, plus two at $d=11$ and six at $d=13$ (in this last case, we do not show the running for the two models with a fermion septet). For a summary of the fields in each model shown here, see appendix B. Continuous lines are for a single copy of each exotic fermion representation, while the dashed lines show the running of $g_{2}$ for three families. In all cases Landau poles appear below the Planck scale, assuming a new physics scale of $2 \mathrm{TeV}$.

three generations, the blow-up of the gauge coupling would occur as low as $10 \mathrm{TeV}$. Finally, we mention that we have checked that in all cases the running of $g_{Y}$ is less strong, than the one we show for $g_{2}$. For this reason, only $g_{2}$ is shown in figure 10.

\subsubsection{Collider physics}

A detailed study of all possible production and decay channels for each of the models, presented above, is beyond the scope of this paper. Instead, here we will concentrate on the most promising signals for the exotic fermions. This choice is motivated by the fact that all models discussed do introduce some exotic fermions, while exotic scalars appear only in some of the models. We will add, however, some comments on exotic scalars at the end of this section.

We have implemented our first $d=9$ model in SARAH [41, 42]. Using Toolbox [43], the implementation can be used to generate SPheno code [44, 45], for the numerical evaluation 

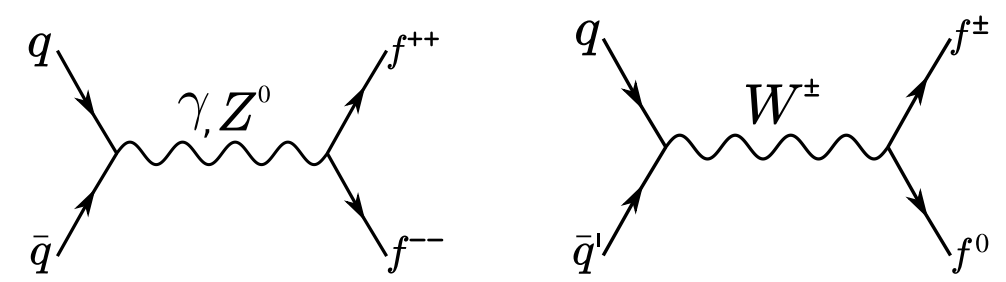

Figure 11. Example Feynman diagrams for the production of exotic fermions appearing in our highdimensional neutrino mass models. Here, $f$ stands symbolically for a fermion from the multiplets $\mathbf{3}_{1}^{F}, \mathbf{4}_{1 / 2}^{F}, \mathbf{4}_{3 / 2}^{F}, \mathbf{5}_{0}^{F}, \mathbf{5}_{1}^{F}, \mathbf{6}_{1 / 2}^{F}, \mathbf{6}_{3 / 2}^{F}$ or $\mathbf{7}_{0}^{F}$.
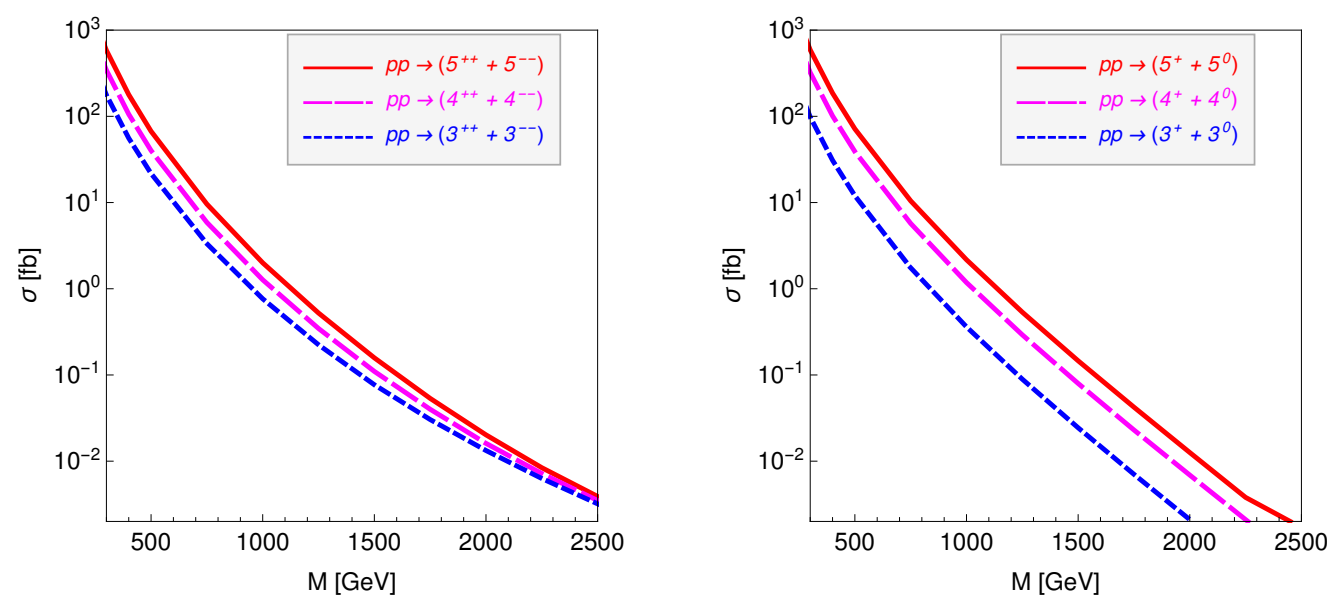

Figure 12. Cross sections for pair production of doubly charged fermions at the LHC for $\sqrt{s}=$ $13 \mathrm{TeV}$, to the left. To the right, associated production of fermions, $f^{+} f^{0}$ for $f$ from $\mathbf{3}_{1}^{F}, \mathbf{4}_{1 / 2}^{F}$ or $\mathbf{5}_{0}^{F}$.

of mass spectra and observables. Production cross sections for the different fermions are then calculated using MadGraph [46].

As we have argued in section 2.3, the new particles of our high-dimensional neutrino mass models should not be heavier than very roughly $m \sim 2 \mathrm{TeV}$, otherwise the treelevel diagram(s) will not give the dominant contribution to the neutrino mass matrix. A natural question to ask then is, whether LHC searches will be able to cover this mass range completely, once sufficient luminosity has been accumulated.

Figure 11 shows some example Feynman diagrams for production of exotic fermions. Here, $f$ stands symbolically for any fermion from the multiplets $\mathbf{3}_{1}^{F}, \mathbf{4}_{1 / 2}^{F}, \mathbf{4}_{3 / 2}^{F}, \mathbf{5}_{0}^{F}, \mathbf{5}_{1}^{F}$, $\mathbf{6}_{1 / 2}^{F}, \mathbf{6}_{3 / 2}^{F}$ or $\mathbf{7}_{0}^{F}$. Numerical example cross sections for pair production of $f^{++} f^{--}$and associated production of $f^{+} f^{0}$ are shown in figure 12 as a function of the fermion mass. These cross sections are for the LHC with a $\sqrt{s}=13 \mathrm{TeV}$. The cross sections are calculated for $\mathbf{3}_{1}^{F}, \mathbf{4}_{1 / 2}^{F}$ and $\mathbf{5}_{0}^{F}$, assuming there is no mixing between the multiplets. Cross sections for fermions from the multiplets $4_{3 / 2}^{F}$ and $5_{1}^{F}$ are slightly larger than the ones shown in the figure. Note that the smallest cross sections are found in all cases for $3_{1}^{F}$.

The plots show that the cross sections for pair production and associated productions are similar for smaller values of the masses, while the pair production cross sections are 

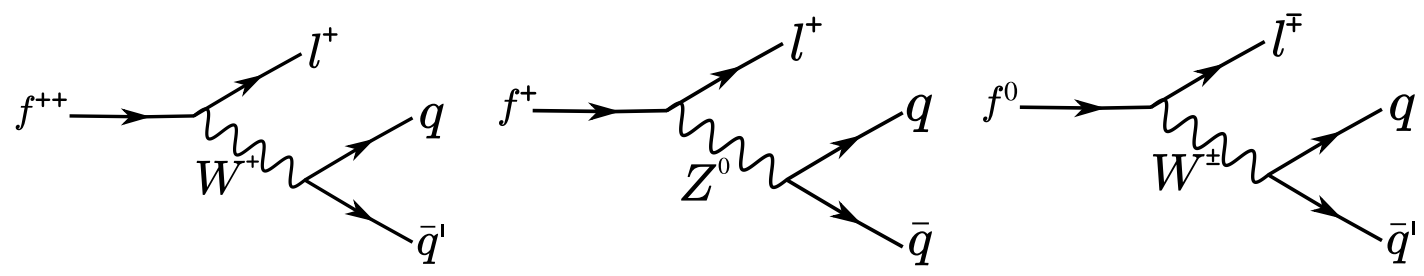

Figure 13. Example Feynman diagrams for the decays of exotic fermions appearing in our highdimensional neutrino mass models.

much larger than associated production for the largest values of masses shown. For this reason, one expects in general more stringent constraints on these fermions will come from searches for pair produced fermions. As figure 12 shows, pair production cross sections are nearly independent of the $\mathrm{SU}(2)_{L}$ quantum numbers for the largest masses. In all cases, the pair production cross section is larger than $10^{-2} \mathrm{fb}$ for masses up to $m=2 \mathrm{TeV}$. Recall that this corresponds to roughly 30 events for the high-luminosity LHC (HL-LHC), albeit before cuts.

A number of different decay channels exist for the doubly charged fermions, in principle. However, the most important decay for the lightest doubly charged fermion is $f^{++} \rightarrow W^{+} l_{\alpha}^{+}$, where $\alpha=e, \mu, \tau$. The relative branching ratios to the different SM lepton generations depends on the unknown Yukawa couplings. The total final state from pairproduced $f^{++} f^{--}$is then either two opposite-sign charged leptons plus four jets, tri-lepton with missing energy plus two jets or multilepton plus missing energy. From the hadronic decays of the $W$ the mass of the doubly charged fermion can be reconstructed. However, multilepton searches might lead to more stringent lower limits on the exotic fermion mass, due to lower backgrounds. The CMS collaboration has recently published a search based on multi-lepton final states [47]. From the analysis presented in [27] one can estimate that this search implies a lower limits on the mass of $f^{++}$of very roughly $[500,750] \mathrm{GeV}$. The range in this interval is due to the large uncertainty in the branching ratio for the different final state lepton generation. Given that the results of [47] are based on $35.9 / \mathrm{fb}$, we expect that the full statistics of the high-luminosity LHC will be sufficient to reach sensitivities up to or in excess of $m \sim 2 \mathrm{TeV}$. To obtain exact numbers would require a MonteCarlo simulation of all backgrounds, which is beyond the scope of this paper.

From the theoretical point of view, however, instead of deriving only limits, it would be more interesting to establish lepton number violation experimentally. For this, we have to turn to associated production. Here, we have to consider the decays of $f^{+}$and $f^{0}$, see figure $11 . f^{+}$can decay to either $l_{\alpha}^{+}+Z^{0}, l_{\alpha}^{+}+h^{0}$ or $\nu+W^{+}$. Since we can not determine experimentally the lepton number in events with missing energy, we are not interested in the final state $\nu+W^{+}$, and only the decays $Z^{0} \rightarrow$ hadrons or $h \rightarrow b \bar{b}$ should be considered when estimating the total number of events. $f^{0}$ can decay to $l_{\alpha}^{ \pm}+W^{\mp}$ and the decay to both charges of leptons should occur with (nearly) equal branching ratios, ${ }^{7}$ as indicated

\footnotetext{
${ }^{7}$ At tree-level $\operatorname{Br}\left(f^{+} \rightarrow l_{\alpha}^{+}+W^{-}\right)=\operatorname{Br}\left(f^{+} \rightarrow l_{\alpha}^{-}+W^{+}\right)$, but for complex Yukawa couplings, small departures from equality are expected to occur at 1-loop level.
} 
in figure 13. The total signal then consists of proton-proton collisions producing the final state $f^{+} f^{0} \rightarrow l_{\alpha}^{+} l_{\beta}^{ \pm}+4 j$, which in the like-sign channel will demonstrate the existence of lepton number violation. If we require 10 events before cuts in both the like-sign and opposite-sign dilepton channels, optimistically we could expect to have sufficient sensitivity at the HL-LHC for masses up to $m \sim 1.6,1.8$ and $2.0 \mathrm{TeV}$ for the triplet, quadruplet and quintuplet fermions. Since this estimate is based only on total cross sections and does not take into account neither cuts nor backgrounds, it should be taken with a grain of salt.

Before closing this section, we want to briefly comment on other fermions, not explicitly covered in this numerical calculation. We have chosen to discuss fermions, because all our models contain at least one new fermion. In fact, $3_{1}^{F}$ appears in the majority of the models that we have discussed. As figure 11 shows for pair production, the most important quantity (apart from the fermion mass) is the fermion charge. Thus, triply charged fermions, such as appear in the multiplets $\mathbf{4}_{3 / 2}^{F}, \mathbf{6}_{1 / 2}^{F}$ and $\mathbf{7}_{0}^{F}$ will have larger cross sections and LHC searches should be able to establish even stronger limits on models containing these particles.

Finally, we briefly comment on the exotic scalars. Scalars have roughly a factor 4 smaller cross sections than fermions with the same mass and quantum numbers. Generally one would thus expect the reach of the LHC to be worse than for fermions. However, the large scalar multiplets that appear in some of our constructions will have highly distinctive final states. An example from pair produced scalars would be 4 charged leptons with $4 W^{\prime}$ 's. Even larger multiplicities can easily occur. Such states should have negligible standard model backgrounds, thus partially compensating for the smaller cross sections. It would be interesting to estimate the LHC reach more quantitatively and we plan to do so in a future publication.

\section{Conclusions}

We have discussed the systematic deconstruction of the $d=9, d=11$ and $d=13$ neutrino mass operators at tree-level. We have found all genuine neutrino mass tree-level diagrams for these operators. The word "genuine" here refers to those diagrams which provide the dominant contribution to the neutrino mass matrix, assuming no extra symmetries beyond the standard model ones. Very few genuine models can be constructed, despite the fact that the number of possible topologies increases rapidly with the dimension of the operator: With renormalizable vertices, one can build 18 topologies and 66 diagrams at $d=9$ level; these numbers increase to 92 topologies and 504 diagrams at the $d=11$ level, and finally at $d=13$ one finds 576 topologies and 4199 diagrams. From all of these, we find only 10 genuine models: 2 models at $d=9$ and $d=11$ each, and 6 models at $d=13$.

We have discussed how our definition of a genuine mass model requires that all these high-dimensional models use large $\mathrm{SU}(2)_{L}$ representations. For example, both of the two $d=9$ models require quadruplets and quintuplets. On the other hand, for some $d=13$ models scalar septets are needed. These high-dimensional models require not only larger representations but also more of them: Three new particles are sufficient for one of the two $d=9$ models, while for $d=11(d=13)$ already four (five) exotic fields are needed. Thus, models become necessarily more baroque with larger dimensions. This fact, together 
with the rather low new physics scale required by the high dimensionality of the operators, makes these models testable at accelerator experiments and also in searches for lepton flavour violation. We therefore plan to return to a study of the phenomenology of these models in a future publication.

\section{Acknowledgments}

We thank Ricardo Cepedello for pointing out to us a misidentified diagram in the first version of this draft. This work was supported by the Spanish grants FPA201785216-P and SEV-2014-0398 (AEI/FEDER, UE), FPU15/03158 (MECD) and PROMETEOII/2018/165 (Generalitat Valenciana). J.C.H. is supported by Chile grants Fondecyt No. 1161463, Conicyt PIA/ACT 1406 and Basal FB0821. O.C-F. is supported by Chile grants Basal FB0821. R.F. also acknowledges the financial support from the Grant Agency of the Czech Republic, (GAČR), contract nr. 17-04902S, as well as from the grant Juan de la Cierva-formación FJCI-2014-21651 (from Spain).

\section{A Neutrino mass and angle fits}

In the main text we gave simple estimates for the typical parameter choices that generate a neutrino mass scale large enough to explain the atmospheric neutrino oscillations. However, in all the models presented in this paper it is actually easy to fit all angles and masses simultaneously. For the current status of oscillation data see, for example, the recently updated global fit [48]. In this appendix we briefly discuss how tree-level neutrino mass models can be fitted to oscillation data.

We can divide all models discussed in the main text into just two classes: (i) models in which only one type of exotic fermion couples to the outside leptons, for example the $d=9$ model shown in figure 6 on the left. And, (ii) models in which two different fermions can couple to the leptons, for example the $d=9$ model in figure 6 on the right.

We start with case (i). First, recall that neutrino oscillations require at least two neutrino masses to be non-zero. For models of case (i) there will be one non-zero neutrino mass for each copy of exotic fermions coupling to the leptons. Assuming there are three copies of these exotic fermions one can then use a slight modification of the well-known Casas-Ibarra parametrization [49] that makes it possible to fit neutrino data for an ordinary seesaw ( $d=5$ tree-level). In the simplest seesaw, the light neutrino mass matrix is approximately given by

$$
m_{\nu}=-m_{D}^{T}\left(\hat{M}_{R}\right)^{-1} m_{D}
$$

where $m_{D}$ is the Dirac mass term for neutrinos and $\hat{M}_{R}$ is the diagonal matrix of the heavy neutrino eigenvalues. Diagonalizing the light neutrino mass matrix with a matrix $V_{L}$ and solving eq. (A.1) for $m_{D}$ one finds [49]

$$
m_{D}=i \sqrt{\hat{M}_{R}} \mathcal{R} \sqrt{\hat{m}_{\nu}} V_{L}^{\dagger}
$$


$\mathcal{R}$ is a matrix of three complex angles, with $\mathcal{R}^{T} \mathcal{R}=1$, left undetermined when solving eq. (A.1). $V_{L}$ contains the measured neutrino angles and Dirac CP-phase $\delta$ and $\hat{m}_{\nu}$ is the diagonal matrix of the light neutrino eigenvalues.

The derivation of eq. (A.2) relies on the fact that in the standard model augmented with a simple seesaw one can always perform a basis change, such that $M_{R}$, the mass matrix for the right-handed neutrinos, is diagonal. In the higher dimensional neutrino mass models, discussed in this paper, for the effective neutrino mass, see eq. (A.1), we have to replace $M_{R}$ by a product of matrices. For example for the $d=9$ model one finds:

$$
M_{R}^{-1} \rightarrow M_{\mathrm{eff}}^{-1}=\hat{M}_{3}^{-1} m_{34}^{T} \hat{M}_{4}^{-1} m_{45}^{T} \hat{M}_{5}^{-1} m_{45} \hat{M}_{4}^{-1} m_{34} \hat{M}_{3}^{-1} .
$$

Basis changes can be used to diagonalize the vector-like mass terms - but not the "Dirac"like mass terms $m_{i j}$ at the same time. $M_{\text {eff }}$ is a complex symmetric matrix and can be diagonalized with a matrix $U$, containing in general 3 angles and 3 phases. For arbitrary choices of the parameters entering the various matrices in eq. (A.3) one can find $U$ numerically and then use eq. (A.2) to determine the correct choice of $m_{D}$, using the simple replacement:

$$
\sqrt{\hat{M}_{R}} \rightarrow U^{\dagger} \sqrt{\hat{M}_{\text {eff }}}
$$

For other models of the same type the form of $M_{\mathrm{eff}}$ may change, but the procedure for the neutrino fit is completely analogous.

For case (ii) one can do a neutrino fit using only one copy of each of the two exotic fermions coupling to leptons. Let $\vec{h}^{1}$ and $\vec{h}^{2}$ be the two Yukawa vectors coupling exotic fermions to standard model leptons in any given model of this type. Then, schematically, one finds a neutrino mass matrix given by:

$$
\left(m_{\nu}\right)_{\alpha \beta}=c\left(h_{\alpha}^{1} h_{\beta}^{2}+h_{\beta}^{1} h_{\alpha}^{2}\right)
$$

Here, $c$ is a constant with dimension of mass. For example, in the $d=9$ model shown on the right of figure $7, c$ is given by $c=Y_{45} \lambda_{4} \frac{\mu_{3} v_{S M}^{6}}{M_{5_{1}} M_{4} m_{4}^{2} m_{3}^{2}}$. The matrix in eq. (A.5) has determinant zero. Thus, it can solved analytically using only quadratic equations. Let $\left|\vec{h}^{i}\right|$ be the absolute value of the vector $\vec{h}^{i}$. Then the two non-zero of eigenvalues eq. (A.5) are given by:

$$
m_{\nu_{2,3}}=c\left(\vec{h}^{1} \cdot \vec{h}^{2} \mp\left|\vec{h}^{1}\right|\left|\vec{h}^{2}\right|\right) .
$$

Neutrino angles, on the other hand, depend only on ratios of entries in the Yukawa vectors. Although also the eigenvectors can be found analytically, neutrino angles are fitted most easily numerically. We calculate $m_{\nu}$ from the measured $\Delta m_{i j}^{2}$ and $\theta_{i j}$. Then, for any choice of the parameters entering $c$, we can choose one entry in the two Yukawa vectors freely, say for example $h_{1}^{1}$, and numerically solve five entries of the matrix in eq. (A.5) for five independent entries in $m_{\nu}$. Note that, since $c$ itself can contain small parameters (for example, all of $Y_{45}, \lambda_{4}$ and $\mu_{3}$ can be small), one needs to check that the resulting Yukawa vectors have entries which are perturbative.

We close this short appendix with a comment. In certain limits, the two types of models can be fitted with both procedures described above. For example, a model in class 
(ii) could have 3 copies of both exotic fermions. If $\vec{h}^{1} \propto \vec{h}^{2}$ for the three pairs of vectors, one can also use the modified Casa-Ibarra procedure to find solutions fitting all data.

\section{B RGEs and $\beta$-coefficients for the different models}

Ignoring the minor contribution from Yukawa couplings, the renormalization group equation for the gauge couplings can be written at 2-loop order as:

$$
\frac{d g_{i}}{d t}=b_{i} \frac{g_{i}^{3}}{(4 \pi)^{2}}+B_{i j} \frac{g_{i}^{3} g_{j}^{2}}{(4 \pi)^{4}}
$$

For the Standard Model, using the ordering $\mathrm{U}(1)_{Y}, \mathrm{SU}(2)_{L}$ and $\mathrm{SU}(3)_{c}$, the coefficients are the following:

$$
b=\left(\frac{41}{10},-\frac{19}{6},-7\right) \text { and } B=\left(\begin{array}{ccc}
\frac{199}{50} & \frac{27}{10} & \frac{44}{5} \\
\frac{9}{10} & \frac{35}{6} & 12 \\
\frac{11}{10} & \frac{9}{2} & -26
\end{array}\right)
$$

In section 3.4.1 we have shown numerical results for the running of $g_{2}$ for six different model variants, two models each at $d=9, d=11$ and $d=13$. Here, for convenience, we repeat the particle content of those six models and give their 1- and 2-loop renormalization group running coefficients (note that those involving $\mathrm{SU}(3)_{c}$, i.e. $b_{3}, B_{3 i}$ and $B_{i 3}$, are the same as in the SM). We considered the cases where the number of copies $n$ of each new fermion representation is either 1 or 3 .

Model-I, $d=9$ (new fields: $3_{ \pm 1}^{F}, 4_{ \pm 1 / 2}^{F}, 5_{0}^{F}$ ):

$$
\begin{array}{llll}
b=\left(\frac{73}{10}, \frac{77}{6},-7\right) & \text { and } & B=\left(\begin{array}{ccc}
\frac{433}{50} & \frac{261}{10} & \frac{44}{5} \\
\frac{87}{10} & \frac{2261}{6} & 12 \\
\frac{11}{10} & \frac{9}{2} & -26
\end{array}\right) & {[n=1]} \\
b=\left(\frac{137}{10}, \frac{269}{6},-7\right) & \text { and } & B=\left(\begin{array}{ccc}
\frac{901}{50} & \frac{729}{10} & \frac{44}{5} \\
\frac{243}{10} & \frac{6713}{6} & 12 \\
\frac{11}{10} & \frac{9}{2} & -26
\end{array}\right) & {[n=3]}
\end{array}
$$

Model-II, $d=9$ (new fields: $4_{3 / 2}^{S}, 5_{ \pm 1}^{F}, 4_{ \pm 1 / 2}^{F}$, real $3_{0}^{S}$ ):

$$
\begin{array}{llll}
b=\left(\frac{107}{10}, \frac{113}{6},-7\right) & \text { and } & B=\left(\begin{array}{ccc}
\frac{407}{10} & \frac{1647}{10} & \frac{44}{5} \\
\frac{549}{10} & \frac{3671}{6} & 12 \\
\frac{11}{10} & \frac{9}{2} & -26
\end{array}\right) & {[n=1]} \\
b=\left(\frac{203}{10}, \frac{353}{6},-7\right) & \text { and } & B=\left(\begin{array}{ccc}
\frac{2791}{50} & \frac{3267}{10} & \frac{44}{5} \\
\frac{1089}{10} & \frac{9851}{6} & 12 \\
\frac{11}{10} & \frac{9}{2} & -26
\end{array}\right) & {[n=3]}
\end{array}
$$


Model-I, $d=11$ (new fields: $3_{ \pm 1}^{F}, 5_{2}^{S}, 5_{1}^{S}$, real $3_{0}^{S}$ ):

$$
\begin{array}{llll}
b=\left(\frac{23}{2}, \frac{13}{2},-7\right) & \text { and } & B=\left(\begin{array}{ccc}
\frac{1307}{10} & \frac{3771}{10} & \frac{44}{5} \\
\frac{1257}{10} & \frac{1129}{2} & 12 \\
\frac{11}{10} & \frac{9}{2} & -26
\end{array}\right) & {[n=1]} \\
b=\left(\frac{163}{10}, \frac{71}{6},-7\right) & \text { and } & B=\left(\begin{array}{ccc}
\frac{6967}{50} & \frac{4059}{10} & \frac{44}{5} \\
\frac{1353}{10} & \frac{3899}{6} & 12 \\
\frac{11}{10} & \frac{9}{2} & -26
\end{array}\right) & {[n=3]}
\end{array}
$$

Model-II, $d=11$ (new fields: $3_{ \pm 1}^{F}, 5_{2}^{S}, 6_{3 / 2}^{S}, 5_{1}^{S}$, real $3_{0}^{S}$ ):

$$
\begin{array}{llll}
b=\left(\frac{71}{5}, \frac{37}{3},-7\right) & \text { and } & B=\left(\begin{array}{ccc}
\frac{4361}{25} & \frac{3303}{5} & \frac{44}{5} \\
\frac{1101}{5} & \frac{3601}{3} & 12 \\
\frac{11}{10} & \frac{9}{2} & -26
\end{array}\right) & {[n=1]} \\
b=\left(19, \frac{53}{3},-7\right) & \text { and } & B=\left(\begin{array}{ccc}
\frac{4577}{25} & \frac{3447}{5} & \frac{44}{5} \\
\frac{1149}{5} & \frac{3857}{3} & 12 \\
\frac{11}{10} & \frac{9}{2} & -26
\end{array}\right) & {[n=3]}
\end{array}
$$

Model-I, $d=13$ (new fields: $3_{ \pm 1}^{F}, 4_{ \pm 3 / 2}^{F}, 5_{ \pm 1}^{F}, 6_{ \pm 1 / 2}^{F}, 5_{0}^{F}$ ):

$$
\begin{array}{llll}
b=\left(\frac{189}{10}, \frac{99}{2},-7\right) & \text { and } & B=\left(\begin{array}{ccc}
\frac{226}{5} & \frac{1008}{5} & \frac{44}{5} \\
\frac{336}{5} & 1596 & 12 \\
\frac{11}{10} & \frac{9}{2} & -26
\end{array}\right) & {[n=1]} \\
b=\left(\frac{97}{2}, \frac{929}{6},-7\right) & \text { and } & B=\left(\begin{array}{ccc}
\frac{3191}{25} & \frac{2997}{5} & \frac{44}{5} \\
\frac{999}{5} & \frac{14329}{3} & 12 \\
\frac{11}{10} & \frac{9}{2} & -26
\end{array}\right) & {[n=3]}
\end{array}
$$

Model-II, $d=13$ (new fields: $4_{3 / 2}^{S}, 5_{ \pm 1}^{F}, 6_{ \pm 1 / 2}^{F}, 5_{0}^{F}$ ):

$$
\begin{array}{llll}
b=\left(\frac{111}{10}, \frac{251}{6},-7\right) & \text { and } & B=\left(\begin{array}{ccc}
\frac{1022}{25} & \frac{936}{5} & \frac{44}{5} \\
\frac{312}{5} & \frac{4480}{3} & 12 \\
\frac{11}{10} & \frac{9}{2} & -26
\end{array}\right) & {[n=1]} \\
b=\left(\frac{43}{2}, \frac{257}{2},-7\right) & \text { and } & B=\left(\begin{array}{ccc}
\frac{1409}{25} & \frac{1971}{5} & \frac{44}{5} \\
\frac{657}{5} & 4305 & 12 \\
\frac{11}{10} & \frac{9}{2} & -26
\end{array}\right) & {[n=3]}
\end{array}
$$

Open Access. This article is distributed under the terms of the Creative Commons Attribution License (CC-BY 4.0), which permits any use, distribution and reproduction in any medium, provided the original author(s) and source are credited.

\section{References}

[1] S. Weinberg, Baryon and lepton nonconserving processes, Phys. Rev. Lett. 43 (1979) 1566 [INSPIRE]. 
[2] P. Minkowski, $\mu \rightarrow e \gamma$ at a rate of one out of $10^{9}$ muon decays?, Phys. Lett. 67B (1977) 421 [INSPIRE].

[3] T. Yanagida, Horizontal symmetry and masses of neutrinos, Conf. Proc. C 7902131 (1979) 95 [INSPIRE].

[4] M. Gell-Mann, P. Ramond and R. Slansky, Complex spinors and unified theories, Conf. Proc. C 790927 (1979) 315 [arXiv:1306.4669] [InSPIRE].

[5] R.N. Mohapatra and G. Senjanović, Neutrino mass and spontaneous parity nonconservation, Phys. Rev. Lett. 44 (1980) 912 [INSPIRE].

[6] M. Magg and C. Wetterich, Neutrino mass problem and gauge hierarchy, Phys. Lett. 94B (1980) 61 [INSPIRE].

[7] J. Schechter and J.W.F. Valle, Neutrino masses in $\mathrm{SU}(2) \times \mathrm{U}(1)$ theories, Phys. Rev. D 22 (1980) 2227 [INSPIRE].

[8] C. Wetterich, Neutrino masses and the scale of B-L violation, Nucl. Phys. B 187 (1981) 343 [INSPIRE].

[9] G. Lazarides, Q. Shafi and C. Wetterich, Proton lifetime and fermion masses in an $\mathrm{SO}(10)$ model, Nucl. Phys. B 181 (1981) 287 [InSPIRE].

[10] R.N. Mohapatra and G. Senjanović, Neutrino masses and mixings in gauge models with spontaneous parity violation, Phys. Rev. D 23 (1981) 165 [INSPIRE].

[11] T.P. Cheng and L.-F. Li, Neutrino masses, mixings and oscillations in $\mathrm{SU}(2) \times \mathrm{U}(1)$ models of electroweak interactions, Phys. Rev. D 22 (1980) 2860 [InSPIRE].

[12] R. Foot, H. Lew, X.G. He and G.C. Joshi, Seesaw neutrino masses induced by a triplet of leptons, Z. Phys. C 44 (1989) 441 [inSPIRE].

[13] F. Bonnet, M. Hirsch, T. Ota and W. Winter, Systematic study of the $d=5$ Weinberg operator at one-loop order, JHEP 07 (2012) 153 [arXiv: 1204.5862] [INSPIRE].

[14] R.N. Mohapatra and J.W.F. Valle, Neutrino mass and baryon number nonconservation in superstring models, Phys. Rev. D 34 (1986) 1642 [INSPIRE].

[15] Particle Data Group collaboration, C. Patrignani et al., Review of Particle Physics, Chin. Phys. C 40 (2016) 100001 [INSPIRE].

[16] Y. Cai, J. Herrero-García, M.A. Schmidt, A. Vicente and R.R. Volkas, From the trees to the forest: a review of radiative neutrino mass models, Front. in Phys. 5 (2017) 63 [arXiv: 1706.08524] [INSPIRE].

[17] A. Zee, A theory of lepton number violation, neutrino Majorana mass and oscillation, Phys. Lett. 93B (1980) 389 [Erratum ibid. B 95 (1980) 461] [INSPIRE].

[18] A. Zee, Quantum numbers of Majorana neutrino masses, Nucl. Phys. B 264 (1986) 99 [INSPIRE].

[19] K.S. Babu, Model of 'calculable' Majorana neutrino masses, Phys. Lett. B 203 (1988) 132 [INSPIRE].

[20] D. Aristizabal Sierra, A. Degee, L. Dorame and M. Hirsch, Systematic classification of two-loop realizations of the Weinberg operator, JHEP 03 (2015) 040 [arXiv:1411.7038] [INSPIRE]. 
[21] L.M. Krauss, S. Nasri and M. Trodden, A model for neutrino masses and dark matter, Phys. Rev. D 67 (2003) 085002 [hep-ph/0210389] [INSPIRE].

[22] M. Gustafsson, J.M. No and M.A. Rivera, Predictive model for radiatively induced neutrino masses and mixings with dark matter, Phys. Rev. Lett. 110 (2013) 211802 [Erratum ibid. 112 (2014) 259902] [arXiv: 1212.4806] [INSPIRE].

[23] R. Cepedello, R.M. Fonseca and M. Hirsch, Systematic classification of three-loop realizations of the Weinberg operator, JHEP 10 (2018) 197 [arXiv:1807.00629] [INSPIRE].

[24] F. Bonnet, D. Hernandez, T. Ota and W. Winter, Neutrino masses from higher than $d=5$ effective operators, JHEP 10 (2009) 076 [arXiv:0907.3143] [INSPIRE].

[25] K.S. Babu, S. Nandi and Z. Tavartkiladze, New mechanism for neutrino mass generation and triply charged Higgs bosons at the LHC, Phys. Rev. D 80 (2009) 071702 [arXiv:0905.2710] [INSPIRE].

[26] R. Cepedello, M. Hirsch and J.C. Helo, Loop neutrino masses from $d=7$ operator, JHEP 07 (2017) 079 [arXiv: 1705.01489] [inSPIRE].

[27] R. Cepedello, M. Hirsch and J.C. Helo, Lepton number violating phenomenology of $d=7$ neutrino mass models, JHEP 01 (2018) 009 [arXiv:1709.03397] [INSPIRE].

[28] I. Picek and B. Radovcic, Novel TeV-scale seesaw mechanism with Dirac mediators, Phys. Lett. B 687 (2010) 338 [arXiv:0911.1374] [INSPIRE].

[29] K. Kumericki, I. Picek and B. Radovcic, TeV-scale seesaw with quintuplet fermions, Phys. Rev. D 86 (2012) 013006 [arXiv:1204.6599] [INSPIRE].

[30] Y. Liao, Cascade seesaw for tiny neutrino mass, JHEP 06 (2011) 098 [arXiv:1011.3633] [INSPIRE].

[31] K.L. McDonald, Minimal tree-level seesaws with a heavy intermediate Fermion, JHEP 07 (2013) 020 [arXiv: 1303.4573] [InSPIRE].

[32] K.L. McDonald, Probing exotic fermions from a seesaw/radiative model at the LHC, JHEP 11 (2013) 131 [arXiv: 1310.0609] [INSPIRE].

[33] T. Nomura and H. Okada, Neutrino mass with large $\mathrm{SU}(2)_{L}$ multiplet fields, Phys. Rev. D 96 (2017) 095017 [arXiv: 1708.03204] [INSPIRE].

[34] T. Nomura, H. Okada and Y. Orikasa, $\mathrm{SU}(2)_{L}$ septet scalar linking to a radiative neutrino model, Phys. Rev. D 94 (2016) 055012 [arXiv: 1605.02601] [InSPIRE].

[35] E. Ma, Pathways to naturally small neutrino masses, Phys. Rev. Lett. 81 (1998) 1171 [hep-ph/9805219] [INSPIRE].

[36] E. Ma, Verifiable radiative seesaw mechanism of neutrino mass and dark matter, Phys. Rev. D 73 (2006) 077301 [hep-ph/0601225] [INSPIRE].

[37] Y. Liao, Unique neutrino mass operator at any mass dimension, Phys. Lett. B 694 (2011) 346 [arXiv: 1009.1692] [INSPIRE].

[38] ATLAS collaboration, Search for doubly-charged Higgs boson production in multi-lepton final states with the ATLAS detector using proton-proton collisions at $\sqrt{s}=13 \mathrm{TeV}$, ATLAS-CONF-2017-053 (2017).

[39] R.C. Read, A survey of graph generation techniques, in Combinatorial Mathematics VIII, K.L. McAvaney ed., Springer (1981). 
[40] R.D. Cameron et al., Cataloguing the graphs on 10 vertices, J. Graph Theory 9 (1985) 551.

[41] F. Staub, SARAH 3.2: Dirac gauginos, UFO output and more, Comput. Phys. Commun. 184 (2013) 1792 [arXiv: 1207.0906] [INSPIRE].

[42] F. Staub, SARAH 4: a tool for (not only SUSY) model builders, Comput. Phys. Commun. 185 (2014) 1773 [arXiv: 1309.7223] [inSPIRE].

[43] F. Staub, T. Ohl, W. Porod and C. Speckner, A tool box for implementing supersymmetric models, Comput. Phys. Commun. 183 (2012) 2165 [arXiv:1109.5147] [INSPIRE].

[44] W. Porod, SPheno, a program for calculating supersymmetric spectra, SUSY particle decays and SUSY particle production at $e^{+} e^{-}$colliders, Comput. Phys. Commun. 153 (2003) 275 [hep-ph/0301101] [INSPIRE].

[45] W. Porod and F. Staub, SPheno 3.1: extensions including flavour, CP-phases and models beyond the MSSM, Comput. Phys. Commun. 183 (2012) 2458 [arXiv:1104.1573] [INSPIRE].

[46] J. Alwall et al., The automated computation of tree-level and next-to-leading order differential cross sections and their matching to parton shower simulations, JHEP 07 (2014) 079 [arXiv: 1405.0301] [INSPIRE].

[47] CMS collaboration, Search for evidence of the type-III seesaw mechanism in multilepton final states in proton-proton collisions at $\sqrt{s}=13$ TeV, Phys. Rev. Lett. 119 (2017) 221802 [arXiv: 1708.07962] [INSPIRE].

[48] P.F. de Salas et al., Status of neutrino oscillations 2018: $3 \sigma$ hint for normal mass ordering and improved CP sensitivity, Phys. Lett. B 782 (2018) 633 [arXiv:1708.01186] [InSPIRE].

[49] J.A. Casas and A. Ibarra, Oscillating neutrinos and $\mu \rightarrow e, \gamma$, Nucl. Phys. B 618 (2001) 171 [hep-ph/0103065] [INSPIRE]. 